\title{
Normal forms and uniform approximations for bridge orbit bifurcations
}

\author{
Ken-ichiro Arita ${ }^{1,2}$ and Matthias Brack ${ }^{2}$ \\ ${ }^{1}$ Department of Physics, Nagoya Institute of Technology, \\ 466-8555 Nagoya, Japan \\ ${ }^{2}$ Institute for Theoretical Physics, University of Regensburg, \\ D-93040 Regensburg, Germany \\ E-mail: arita@nitech.ac.jp, Matthias.Brack@physik.uni-regensburg.de
}

\begin{abstract}
We discuss various bifurcation problems in which two isolated periodic orbits exchange periodic "bridge" orbit(s) between two successive bifurcations. We propose normal forms which locally describe the corresponding fixed point scenarios on the Poincare surface of section. Uniform approximations for the density of states for an integrable Hamiltonian system with two degrees of freedom are derived and successfully reproduce the numerical quantummechanical results.

PACS numbers: $05.45 . \mathrm{Mt}$, 03.65.Sq
\end{abstract}

\section{Introduction}

The periodic orbit theory [1, 2, 3] has made important contributions to the understanding of quantum chaos [4, 5, 6] and to the semiclassical interpretation of quantum shell effects in finite fermion systems [7. Through semiclassical trace formulae, it relates the density of states of a quantum Hamiltonian to the sum over all periodic orbits of the corresponding classical Hamiltonian system. Gutzwiller's trace formula [1] assumes the periodic orbits to be isolated and therefore applies most directly to chaotic systems; it can, however, also be used for integrable systems with isolated orbits (see e.g., [8, 9]).

In the derivation of the trace formula [1, the stationary phase approximation is used for evaluating some of the trace integrals over the semiclassical amplitude, leading to Gauss-Fresnel integrals. In systems with regular or mixed classical dynamics, periodic orbits can undergo bifurcations at critical values of a system parameter (e.g., energy or deformation). At such critical points, one or more of the Gauss-Fresnel integrals become singular and cause the divergence of the Gutzwiller amplitudes of the bifurcating orbits. This situation can be remedied [10] by going to higher than second-order terms in the expansion of the action function, which appears in the phase of the trace integrand, around the critical point in phase space. The minimum number of terms required in this expansion are given by the so-called normal forms, which are characteristic for each type of bifurcation (see also [11]) and lead to usually wellknown catastrophe integrals. At the bifurcation points, one obtains in this way local uniform approximations for the semiclassical amplitudes that are finite and contain the contributions of all orbits involved in the bifurcation. 
The local uniform approximations do, however, not reproduce the correct asymptotic Gutzwiller amplitudes far away from the bifurcation point where all involved orbits are isolated. To achieve this goal, global uniform approximations must be developed which interpolate smoothly from the local behavior at the bifurcation to the asymptotic regions of the isolated orbits. This has been done in Refs. 13, 14, 15, for all generic bifurcations occurring in Hamiltonian systems with two degrees of freedom according to the classification of Meyer [12] (and listed also in [11), in Refs. [16, 17] for codimension-two bifurcations, and in Ref. [18 for the transcritical bifurcation. (In passing, we mention that similar divergences of the Gutzwiller amplitudes occur when a symmetry is broken - or restored - under the variation of a system parameter. Local uniform approximations for symmetry breaking have been developed in Refs. [10, 19]; the prototype of a global uniform approximation for the breaking of U(1) symmetry was developed in Ref. [20, which inspired those mentioned above for bifurcations as well as global uniform approximations for the breaking of other symmetries 21.)

In this paper we investigate a type of bifurcation that has not yet been studied in this context and that we term bridge orbit bifurcation. Typically, this bifurcation consists of a pair of isolated orbits which are connected through a "bridge orbit" that only exists in a finite interval of the system parameter. Under its monotonous variation, the bridge orbit is born at a bifurcation of the first isolated orbit and then absorbed at a bifurcation of the second isolated orbit. This scenario has been found in both integrable and non-integrable Hamiltonian systems 22, 23, 24]. It occurs, e.g., in the two-dimensional rationally deformed harmonic oscillator under a generic class of perturbations; other examples will be given in section 2 In the integrable case the bridge orbit forms a continuously degenerate family (i.e., a rational torus), while in non-integrable cases it is typically isolated. Since the two isolated orbits which exchange the bridge orbit typically are well separated in phase space, the bridge bifurcation is accompanied by global changes of the phase space structure which cannot be treated with the usual perturbative normal forms derived from the BirkoffGustavson expansion. This is different from the generic bifurcations of codimension one [11, and also from those of codimension two considered in [16, 17, where only local changes in the phase space structure occur around a central periodic orbit that exists at all values of the relevant system parameter.

In all the bifurcation types investigated so far in connection with uniform approximations, all orbits participating in the bifurcations become asymptotically isolated far enough from the bifurcation point(s). Even in the codimension-two bifurcations considered in [16, 17, where some isolated orbits only exist between two adjacent bifurcations, these have their well-defined partners which become asymptotically isolated at least on one side of one of the bifurcations. For the bridge orbits studied here, this is not the case. The bridge orbits are entirely intrinsic to the bifurcation scenario and cannot be linked to any 'external' orbit existing far away from the bifurcation. This causes a generic problem in the construction of the global uniform approximations which will be discussed in section 4 .

In section 2, we present some examples of bridge orbit bifurcations in integrable and non-integrable systems with two degrees of freedom. In section 3. we propose a new type of normal form (derived in detail in the appendix) which successfully describes the scenario of the bridge orbit bifurcation in integrable systems. In section 4, we derive local and global uniform approximations from this normal form, give analytic trace formulae for the semiclassical density of states and compare its numerically computed results with fully quantum-mechanical results for the 
corresponding quantum Hamiltonians. Section [5] is devoted to a summary and concluding remarks.

In the following sections, numerical integrations of the classical equations of motion are performed with Adams' Method (a kind of predictor-corrector method) to get solutions of high accuracy. Periodic orbits are obtained by searching fixed points $(q, p)$ of the Poincaré map in a suitable surface of section using a two-dimensional Newton-Raphson iteration method, whereby the stability matrix (linearized Poincaré map) is obtained at the same time. The fixed points are smooth functions of the system parameter and can easily be followed through the bifurcation points under variation of the parameter, as well as new branches of fixed points emerging from the bifurcation points.

\section{Bridge orbit bifurcations in two dimensional $r^{\alpha}$ potential models}

Let us start from a system with two degrees of freedom described by the Hamiltonian

$$
H_{0}(\mathbf{p}, \mathbf{r})=\frac{1}{2} \mathbf{p}^{2}+\frac{1}{2}|\mathbf{r}|^{\alpha} \text {. }
$$

Since the potential is a homogeneous function of the coordinates $\mathbf{r}=(x, y)$, the Hamiltonian has the scaling property

$$
H_{0}\left(c^{\frac{1}{2}} \mathbf{p}, c^{\frac{1}{\alpha}} \mathbf{r}\right)=c H_{0}(\mathbf{p}, \mathbf{r}), \quad c>0,
$$

and the equations of motion $(\mathrm{EOM})$ are invariant under the following scaling transformation:

$$
\mathbf{p} \rightarrow c^{\frac{1}{2}} \mathbf{p}, \quad \mathbf{r} \rightarrow c^{\frac{1}{\alpha}} \mathbf{r}, \quad t \rightarrow c^{\frac{1}{\alpha}-\frac{1}{2}} t,
$$

while the energy transforms as $E \rightarrow c E$. Therefore, the phase-space profile is independent of energy and one has the same set of periodic orbits at all energies $E$. The action integral along a periodic orbit (po) has the following energy dependence

$$
S_{\mathrm{po}}(E)=\oint_{\mathrm{po}(E)} \mathbf{p} \cdot \mathrm{d} \mathbf{r}=\left(\frac{E}{E_{0}}\right)^{\frac{1}{2}+\frac{1}{\alpha}} \oint_{\mathrm{po}\left(E_{0}\right)} \mathbf{p} \cdot \mathrm{d} \mathbf{r}=\hbar \mathcal{E} \tau_{\mathrm{po}},
$$

where the dimensionless scaled energy $\mathcal{E}$ and the dimensionless scaled period $\tau_{\text {po }}$ of the orbit are defined by

$$
\mathcal{E}=\left(\frac{E}{E_{0}}\right)^{\frac{1}{2}+\frac{1}{\alpha}}, \quad \tau_{\mathrm{po}}=\frac{1}{\hbar} \frac{\partial S_{\mathrm{po}}}{\partial \mathcal{E}}=\frac{1}{\hbar} \oint_{\mathrm{po}\left(E_{0}\right)} \mathbf{p} \cdot \mathrm{d} \mathbf{r},
$$

whereby the reference energy $E_{0}$ can be chosen arbitrarily.

We now consider two kinds of perturbations of the system (2.1). The first one is introduced by a magnetic field perpendicular to the $(x, y)$ plane. The motion of a charged particle in the plane is described by the Hamiltonian

$$
H_{\kappa}=\frac{1}{2} \mathbf{p}^{2}+\frac{1}{2} r^{\alpha}-\kappa r^{\frac{\alpha}{2}-1}\left(x p_{y}-y p_{x}\right)
$$

where the radial dependence of the perturbation (with $r=|\mathbf{r}|$ ) is determined such that the scaling invariance persists for any finite $\kappa$. The strength of the magnetic field is proportional to $\kappa r^{\frac{\alpha}{2}-1}$. Using polar coordinates $\mathbf{r}=(r, \theta), \mathbf{p}=\left(p_{r}, p_{\theta}\right)$, (2.6) reads

$$
H_{\kappa}=\frac{1}{2}\left(p_{r}^{2}+\frac{p_{\theta}^{2}}{r^{2}}\right)+\frac{1}{2} r^{\alpha}-\kappa r^{\frac{\alpha}{2}-1} p_{\theta} .
$$




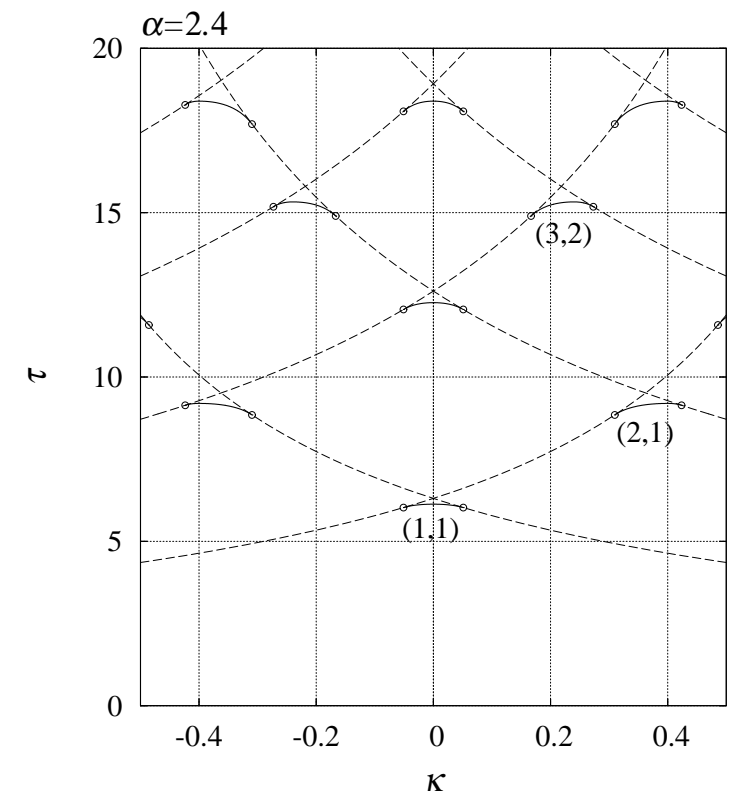

Figure 1. Scaled periods $\tau$ of periodic orbits for the Hamiltonian (2.6) as functions of $\kappa$. Isolated circular orbits and families of bridge orbits are indicated by dashed and solid curves, respectively. Circles represent bifurcation points.
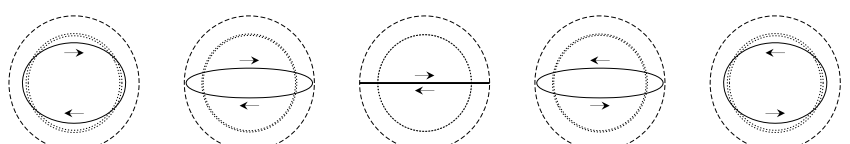

$\kappa=-0.05$

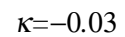

$\kappa=0$

$\kappa=0.03$

$\kappa=0.05$
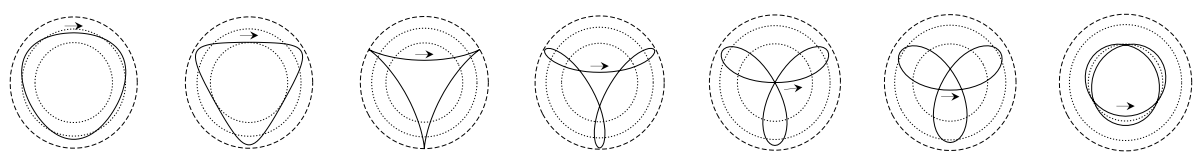

$\kappa=0.314$

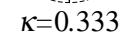

$\kappa=0.36$

$\kappa=0.4$

$\kappa=0.41$

$\kappa=0.4234$
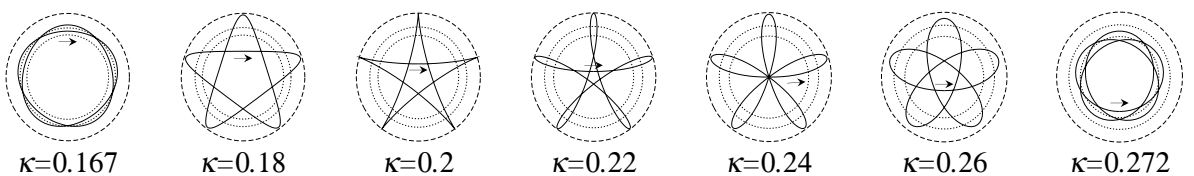

Figure 2. Some short bridge orbits in Hamiltonian (2.6) for $\alpha=2.4$ and for different values of $\kappa$. Upper row shows symmetric $(1,1)$ bridge, middle and lower rows show asymmetric $(2,1)$ and $(3,2)$ bridges, respectively. 
This system is integrable since $p_{\theta}=\Lambda$ is a constant of motion. For any non-zero $\kappa$, there exist two isolated circular periodic orbits $\mathrm{C}_{ \pm}$with different radii $r_{ \pm}$and angular momenta $\Lambda_{ \pm}$, which are found as solutions of the equations

$$
\begin{aligned}
& V_{\text {eff }}(r, \Lambda)=\frac{1}{2} r^{\alpha}+\frac{\Lambda^{2}}{2 r^{2}}-\kappa \Lambda r^{\frac{\alpha}{2}-1}=E, \\
& \frac{\partial V_{\text {eff }}}{\partial r}(r, \Lambda)=\frac{\alpha}{2} r^{\alpha-1}-\frac{\Lambda^{2}}{r^{3}}-\kappa \Lambda\left(\frac{\alpha}{2}-1\right) r^{\frac{\alpha}{2}-2}=0 .
\end{aligned}
$$

Evidently they depend on the parameter $\kappa$. The radii $r_{ \pm}$correspond simply to the minima of the effective potential (2.8). Small oscillations around the circular orbits $\mathrm{C}_{ \pm}$have frequencies $\omega_{ \pm}$for their angular and $\Omega_{ \pm}$for their radial motions which are given by

$$
\omega_{ \pm}=\frac{\Lambda_{ \pm}}{r_{ \pm}^{2}}-\kappa r_{ \pm}^{\frac{\alpha}{2}-1}, \quad \Omega_{ \pm}=\sqrt{\frac{\partial^{2} V_{\mathrm{eff}}}{\partial r^{2}}\left(r_{ \pm}, \Lambda_{ \pm}\right)} .
$$

They become periodic for those values $\kappa_{ \pm}$for which the two frequencies are commensurate, i.e., when

$$
\frac{\Omega_{ \pm}}{\omega_{ \pm}}\left(\kappa_{ \pm}\right)= \pm \frac{\left(n_{+}+n_{-}\right)}{n_{ \pm}}
$$

with positive integers $n_{+}$and $n_{-}$. Precisely at the values $\kappa=\kappa_{ \pm}$, the orbits $\mathrm{C}_{ \pm}$ must undergo bifurcations, because the trace of their stability matrix $M$ equals two: $\operatorname{Tr} M_{\mathrm{C}_{ \pm}}\left(\kappa_{ \pm}\right)=+2$. The interesting phenomenon now is that the two bifurcations are connected by a bridge orbit $\mathrm{B}$ that is created (or absorbed) at the bifurcations. It is actually a degenerate family of orbits with $\operatorname{Tr} M_{\mathrm{B}}(\kappa)=+2$ for all values $\kappa_{-} \leq \kappa \leq \kappa_{+}$. More precisely, a bridge orbit $\mathrm{B}$ emerges from a bifurcation of the $n_{-}$-th repetition of the orbit $\mathrm{C}_{-}$at $\kappa=\kappa_{-}$and is absorbed at a bifurcation of the $n_{+}$-th repetition of the orbit $\mathrm{C}_{+}$at $\kappa=\kappa_{+}$. It can therefore be labeled by the repetition numbers as $\mathrm{B}\left(n_{+}, n_{-}\right)$. Figure 1 shows the scaled periods of the shortest periodic orbits of the system (2.6) as functions of the parameter $\kappa$. At each crossing point of some repetitions of the two circular orbits, indicated by the pair of numbers $\left(n_{+}, n_{-}\right)$, one finds a bridge orbit family connecting them. The shapes of some of the bridge orbits are shown in figure 2. In figure 3, the scaled periods and the traces of stability matrices are plotted as functions of $\kappa$ for the circular orbits $\mathrm{C}_{ \pm}$and the bridge orbits connecting them. $\operatorname{Tr} M_{\mathrm{C}_{ \pm}}(\kappa)$ touches the horizontal line $\operatorname{Tr} M=2$ at $\kappa=\kappa_{ \pm}$, and in between there exist the bridge orbit families having $\operatorname{Tr} M_{\mathrm{B}}=2$.

The second perturbation is introduced by an elliptic deformation. We modify the Hamiltonian (2.11) as follows:

$$
\begin{aligned}
& H_{\beta}=\frac{1}{2} \mathbf{p}^{2}+\frac{1}{2}\left(r f_{\beta}(\theta)\right)^{\alpha}, \\
& f_{\beta}(\theta)=\sqrt{\eta \cos ^{2} \theta+\frac{1}{\eta} \sin ^{2} \theta}, \quad \beta=\frac{2(\eta-1)}{\eta+1} .
\end{aligned}
$$

The scaling rule (2.2) persists for any $\beta$, but the system is nonintegrable for $\beta \neq 0$. We take $\beta$ as the deformation parameter, which is related to the axis ratio $\eta$ by the second equation in (2.13). For any $\beta \neq 0$, there are two isolated periodic orbits $\mathrm{A}_{x}$ and $\mathrm{A}_{y}$ : straight-line librations along the $x$ and $y$ axis, respectively. Figure 4 shows the scaled periods of the shortest periodic orbits in the system (2.12) as functions of $\beta$. Again one, finds bridge orbits $\mathrm{B}$ which bifurcate from the repetitions $\left(n_{x}, n_{y}\right)$ of the orbits $\mathrm{A}_{x}$ and $\mathrm{A}_{y}$ near each crossing point. 

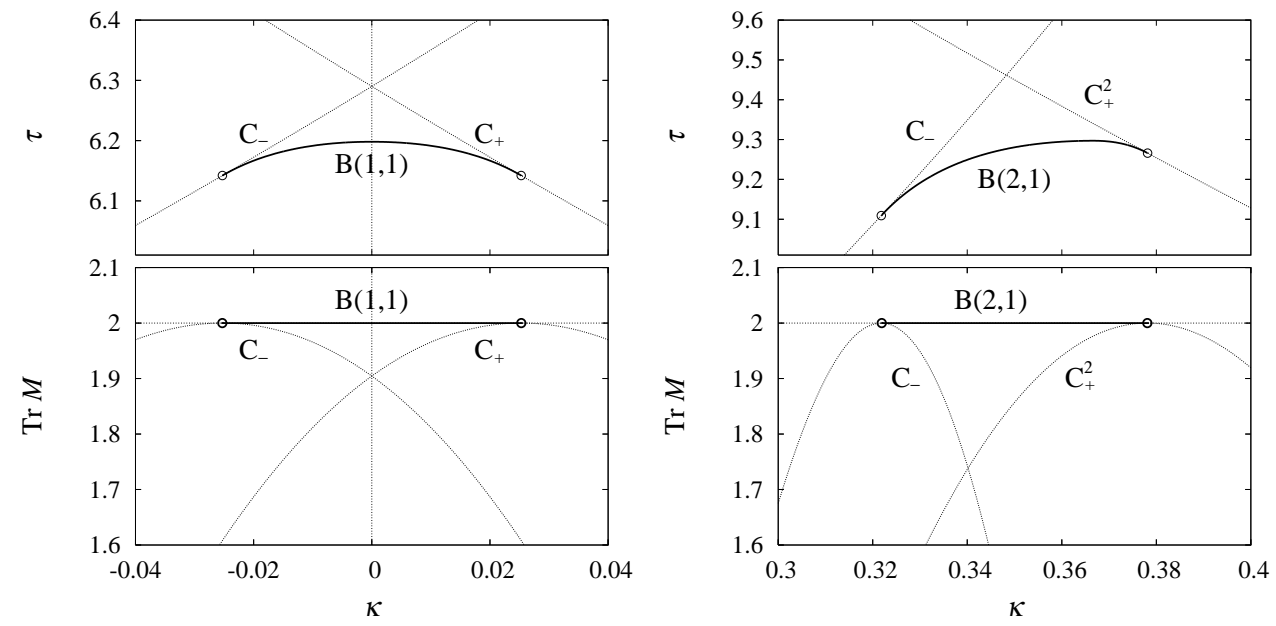

Figure 3. Scaled period $\tau$ (upper panels) and trace of stability matrix $M$ (lower panels) of the symmetric $(1,1)$ (left-hand side) and asymmetric $(2,1)$ (right-hand side) bridge orbits for the Hamiltonian (2.6) with $\alpha=2.2$, shown by solid lines as functions of the parameter $\kappa$. The dashed lines show the corresponding quantities for the circular orbits $\mathrm{C}_{ \pm}$.

In figure 5 we plot some of the shortest bridge orbits in the $(x, y)$ plane. The system (2.12) is non-integrable and the bridge orbits here are isolated. The symmetric bridges $\mathrm{B}(m, m)$ are bounded by two isochronous pitchfork bifurcations; the example of $\mathrm{B}(1,1)$ is illustrated in figure 6. In the left panel of figure 7 , the graph of $\operatorname{Tr} M(\beta)$ for the 2 nd repetition of the $\mathrm{A}_{x}$ orbit touches the horizontal line $\operatorname{Tr} M=+2$ at $\kappa=0.628$ and two stable and unstable branches emerge from an island-chain bifurcation. The unstable and stable branches submerge sequentially into the $\mathrm{A}_{y}$ orbit at $\kappa=0.688$ and 0.728 , respectively, in two successive pitchfork bifurcations. For asymmetric crossings $\left(n_{x}, n_{y}\right)$ with $n_{x} \neq n_{y}$, the bridges consist of two isolated branches, i.e., one stable and one unstable orbit, which we label by $\mathrm{B}\left(n_{x}, n_{y}\right)_{s}$ and $\mathrm{B}\left(n_{x}, n_{y}\right)_{u}$, respectively. For the bridge orbits $\mathrm{B}(2,1)$, the common left end is a non-generic island-chain bifurcation of the 2 nd repetition of the $\mathrm{A}_{x}$ orbit, while the right ends are two isochronous pitchfork bifurcations of the $\mathrm{A}_{y}$ orbit occurring at two different deformations (see the left panel of figure (7). The asymmetric $\mathrm{B}\left(n_{+}, n_{-}\right)$bridges with $n_{+}, n_{-} \geq 2$ also consist of two isolated branches with different actions and stabilities, but their end points are common at both ends of both branches, since they occur both at period-multiplying island-chain bifurcations (cf. the right panel in figure 7 for the $\mathrm{B}(3,2)$ bridges).

In the limit $\alpha \rightarrow 2, H_{\kappa}$ becomes the cranked and $H_{\beta}$ is the anisotropic harmonic oscillator. (Note that both systems have identical spectra after a suitable transformation from $\alpha$ to $\kappa$, cf. section 3.2.8 of (7)). In this limit, each bifurcation pair coalesces and the connecting bridge orbit shrinks to a point at which the two isolated orbits intersect. At the crossing points, one has locally periodic-orbit families of twofold degeneracy due to the dynamical SU(2) symmetry of the rationally deformed harmonic oscillator in two dimensions.

A scenario involving two bridge orbits is obtained if one breaks the U(1) symmetry that the Hamiltonian (2.13) possesses at $\beta=0$. Let us e.g., modify the shape 


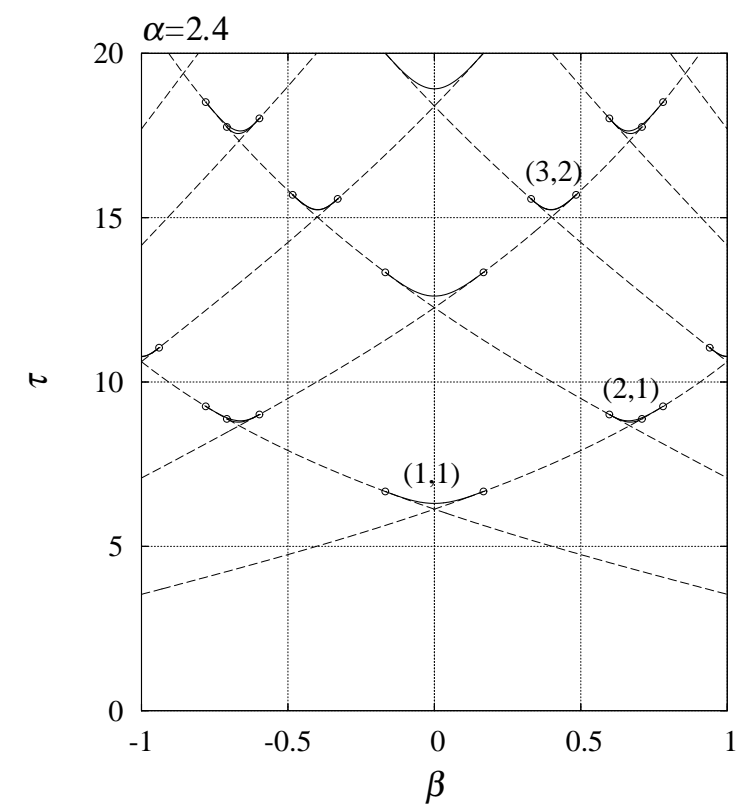

Figure 4. Same as figure 1 but for the Hamiltonian (2.12) as functions of deformation parameter $\beta$. Bridge orbits are isolated in contrast to those for (2.6).
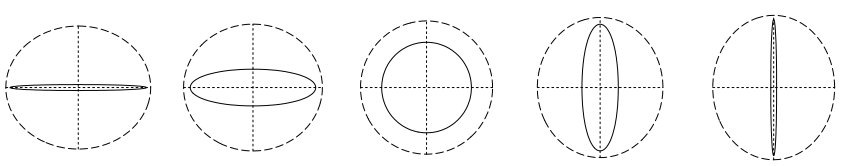

$\beta=-0.16$

$\beta=-0.1$

$\beta=0.0$

$\beta=0.1$

$\beta=0.16$
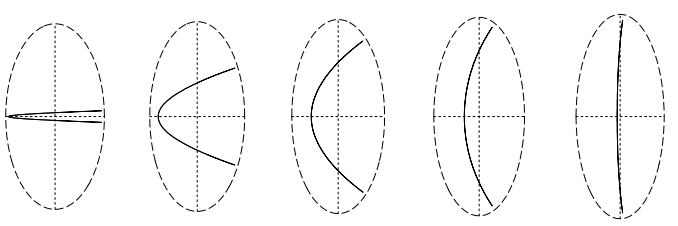

$\beta=0.6$

$\beta=0.64$

$\beta=0.68$

$\beta=0.72 \quad \beta=0.77$
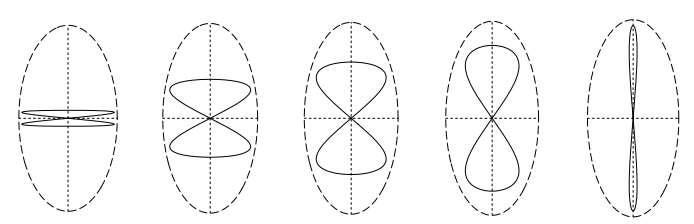

$\beta=0.604$

$\beta=0.64$

$\beta=0.66$

$\beta=0.68 \quad \beta=0.708$

Figure 5. Some short bridge orbits in the Hamiltonian (2.6) for $\alpha=2.4$ and for several values of deformation parameters $\beta$. The upper row shows the symmetric bridge $\mathrm{B}(1,1)$, the middle and lower rows display the stable and unstable branches of the asymmetric $\mathrm{B}(2,1)$ bridge. 


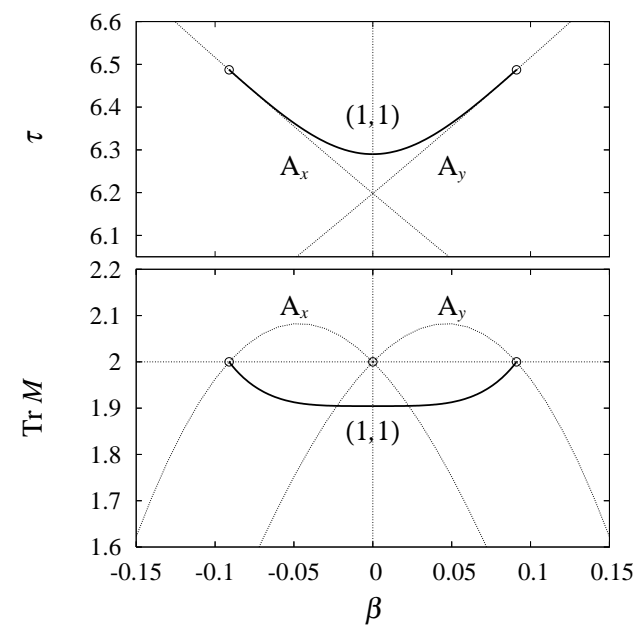

Figure 6. Scaled period $\tau$ (upper panel) and trace of the stability matrix $M$ (lower panel) of the symmetric $(1,1)$ bridge orbit for the Hamiltonian (2.12) with $\alpha=2.2$, shown by solid lines as functions of the deformation parameter $\beta$. The dashed lines show the corresponding quantities for the $\mathrm{A}_{x}$ and $\mathrm{A}_{y}$ orbits.
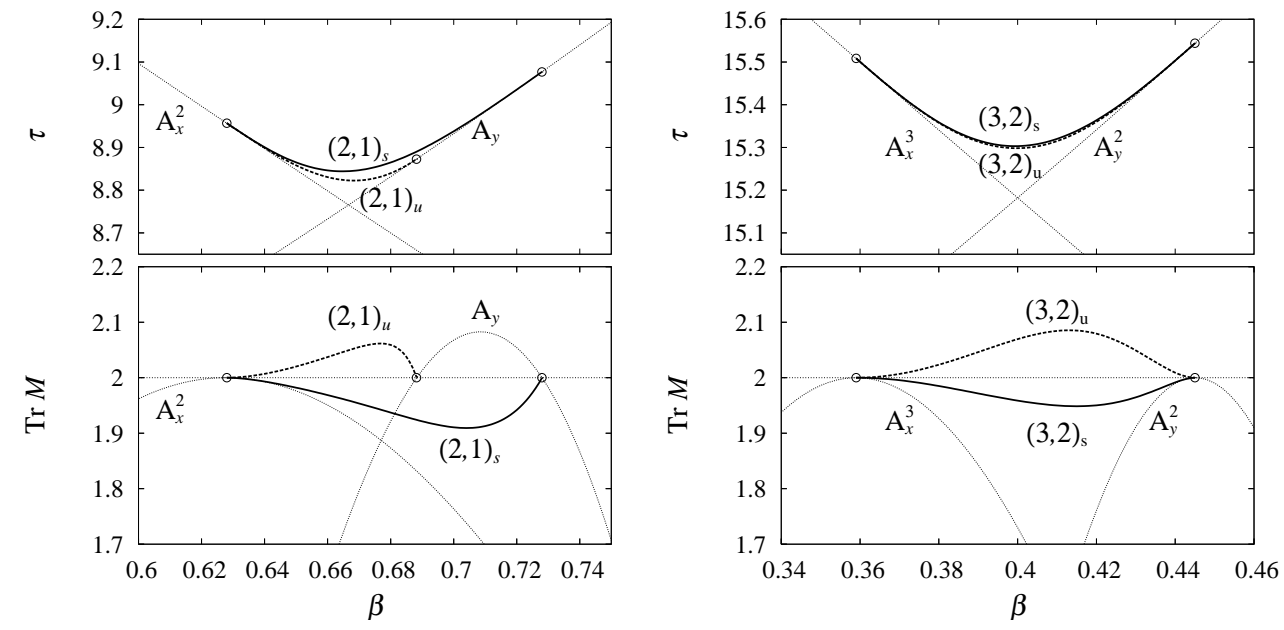

Figure 7. Same as figure 6 but for asymmetric $\left(n_{x}, n_{y}\right)$ bridge orbit bifurcations, with two pairs of values for the $n_{x}$-th repetition of the $\mathrm{A}_{x}$ orbit the and the $n_{y}$-th repetition of the $\mathrm{A}_{y}$ orbit. The suffixes $s$ and $u$ represent the stable and unstable branches, respectively. 

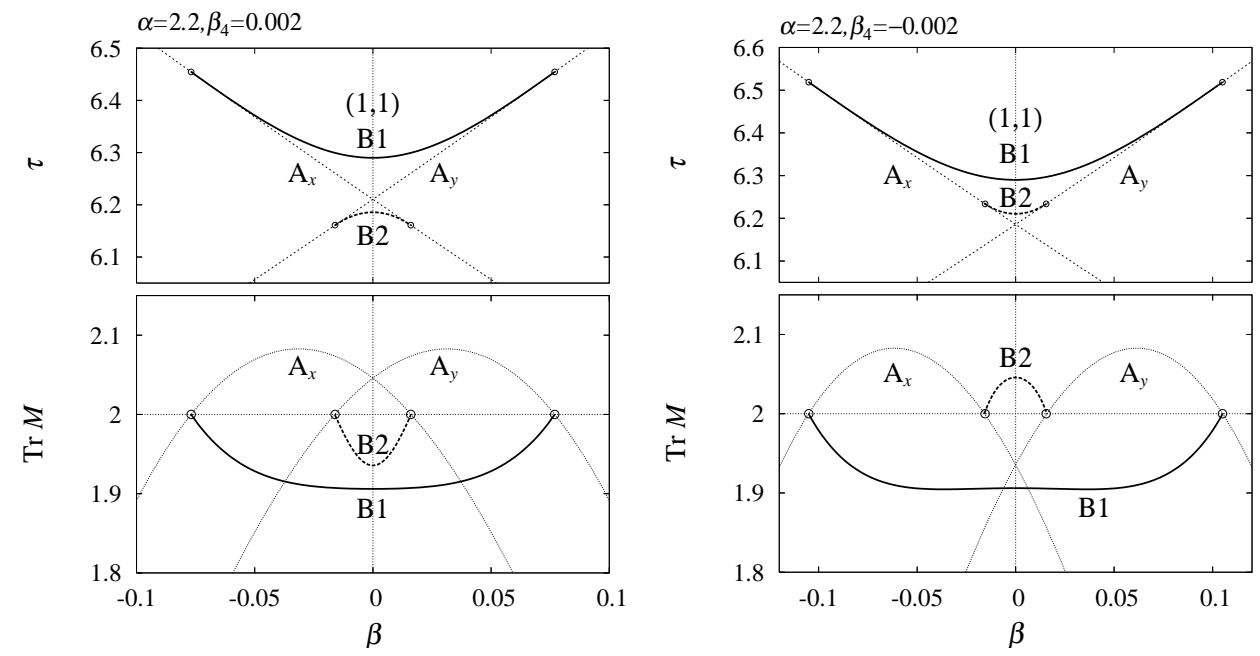

Figure 8. Same as figure 6 but for the shape function (2.14) with $\beta_{4}= \pm 0.002$ and $\alpha=2.2$.

function $f_{\beta}(\theta)$ in (2.13) by the following one:

$$
f_{\beta, \beta_{4}}(\theta)=\sqrt{\eta \cos ^{2} \theta+\frac{1}{\eta} \sin ^{2} \theta}-\beta_{4} \cos 4 \theta,
$$

with nonzero $\beta_{4}$. In this case, a second bridge orbit appears around $\beta=0$ for the symmetric $(m, m)$ bifurcations. Figure 8 shows the properties of these bridge orbits. For $\beta_{4}=0$ it corresponds to figure 6 where the two isolated orbits $\mathrm{A}_{x}$ and $\mathrm{A}_{y}$ intersect in a point with $\mathrm{U}(1)$ symmetry $(\beta=0)$ and their stability traces intersect at $\operatorname{Tr} M=2$ (see the lower part of the figure). For $\beta_{4} \neq 0$, this crossing point is split, so that the second bifurcation of each orbit occurs at different points on the $\beta$ axis; the new pair of bifurcations is now connected by a second bridge orbit B2. For $\beta_{4}>0$, as shown on the left side of figure 8 both bridges are stable, while for $\beta_{4}<0$ (see right side) one of them is stable and the other is unstable. All bifurcations here are of the non-generic pitchfork type.

Finally, we mention a bifurcation scenario that has been discussed in [24. Hereby a pair of isolated orbits exchange their stability via an isolated bridge orbit. Examples for this are found in the coupled quartic oscillator

$$
H_{\alpha}=\frac{1}{2} \mathbf{p}^{2}+\frac{1}{4}\left(x^{4}+y^{4}\right)+\alpha x^{2} y^{2} .
$$

Figure 9 shows a narrow region of the chaoticity parameter $\alpha$. The shapes of two crossing isolated orbits $\mathrm{F}$ and $\mathrm{P}$ in the $(x, y)$ plane are shown by inserts, as well as various shapes of the bridge orbit $\mathrm{Q}$ interpolating between those two shapes. Note the extremely small scale: the maximum value of $|\operatorname{Tr} M-2|$ of the bridge orbit is smaller than $10^{-7}$. On a larger scale, the bridge orbit may not be observed numerically and the two isolated orbits $\mathrm{F}$ and $\mathrm{P}$ would appear to cross in a point. The bifurcation diagram then would look similar to that of a transcritical bifurcation [18. The orbits $\mathrm{F}$ and $\mathrm{P}$ are created at $\alpha=0.6315$ in a period-tripling bifurcation from a straight-line libration along the $y$ axis; at $\alpha=0$ they become members of an integrable 3:2 torus 


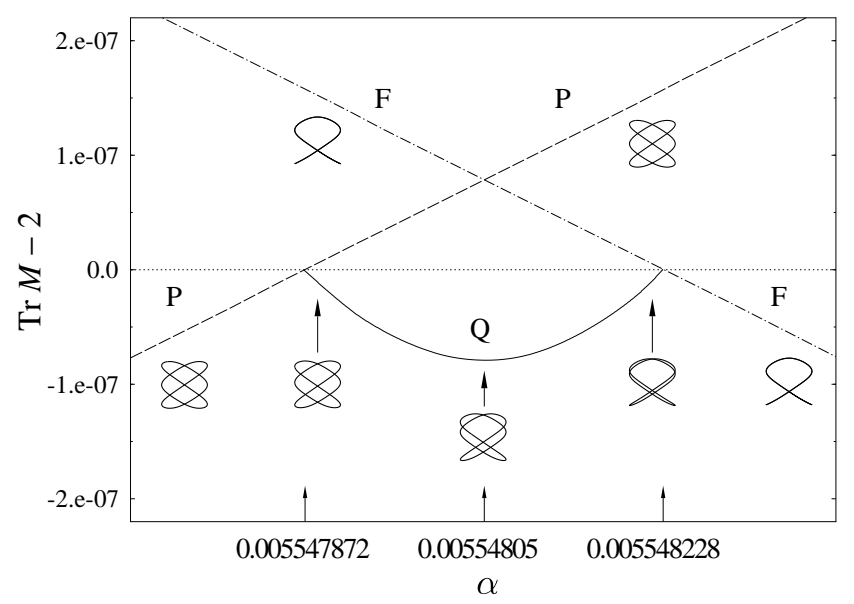

Figure 9. Stability exchange of isolated orbits $\mathrm{F}$ and $\mathrm{P}$ in the coupled quartic oscillator (2.15) via two non-generic pitchfork bifurcations connected by an isolated bridge orbit $\mathrm{Q}$. The inserts exhibit the shapes of the orbits in the $(x, y)$ plane.

with $\mathrm{U}(1)$ symmetry. We refer to [18 for details of this bifurcation scenario and to 24, 25] for details of the potential (2.15). A bridge bifurcation of the same type has been also found to occur in a two-dimensional spin-boson Hamiltonian [26].

\section{Normal forms for some bridge-orbit bifurcation scenarios}

Normal forms are frequently used in singularity theory (see e.g., [27]) and catastrophe theory (see e.g., 28, to classify bifurcations. The natural variables of the normal forms for bifurcations in Hamiltonian systems with two degrees of freedom are the two canonical variables $(q, p)$ spanning a projected Poincaré surface of section transverse to the bifurcating parent orbit, and a bifurcation parameter $\epsilon$. These variables are usually chosen such that the bifurcation of the parent orbit occurs at $(q, p, \epsilon)=(0,0,0)$. The normal form function $S(q, p, \epsilon)$ must fulfill the condition that its critical points correspond to the fixed points in $(q, p, \epsilon)$ space in the neighborhood of the origin, and hence to the periodic orbits taking part in the bifurcation. This condition, however, is not sufficient to specify the normal form for a given bifurcation uniquely. Usually, $S(q, p, \epsilon)$ is chosen to contain a minimum number of parameters and simple functions of the variables $(q, p, \epsilon)$ - often just polynomial expressions in $q, p$ and $\epsilon$ - that yield the desired fixed-point scenario of a given bifurcation. For the generic bifurcations in two-dimensional symplectic maps according to the classification of Meyer [12, the standard normal forms have been given in [10, 11]. They can also be used for nongeneric bifurcations of the same type (i.e., with the same fixed-point scenario in phase space) occurring in systems with discrete symmetries (see e.g., [29]). Normal forms for non-generic bifurcations in Hamiltonian systems with different fixed-point scenarios can be found in [18] (for the transcritical bifurcation) and in [16, 17] (for codimensiontwo bifurcations).

For bifurcations involving more than two orbits, it is often useful to transform 


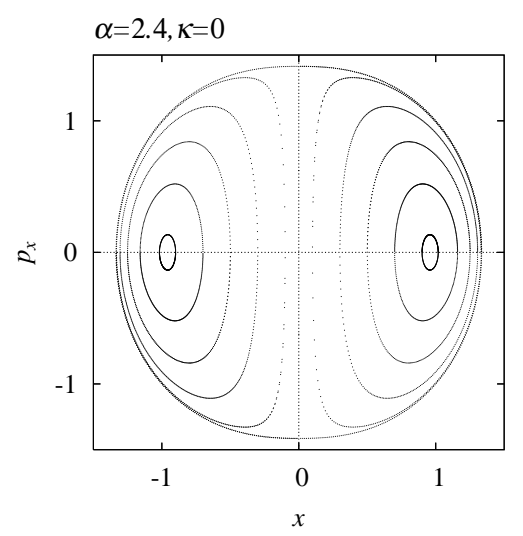

Figure 10. Poincaré surface of section for (2.6) with $\alpha=2.4$ and $\kappa=0$.

the Poincaré variables $(q, p)$ to action-angle variables $(\varphi, I)$ (cf. [11, 15]):

$$
p=\sqrt{2 I} \cos \varphi, \quad q=\sqrt{2 I} \sin \varphi, \quad I \geq 0, \quad \varphi \in[0,2 \pi) .
$$

This becomes particularly useful when one considers integrable systems for which the normal form function does not depend on the angle $\varphi$, so that one only has to deal with a function $S(I, \epsilon)$ depending on two variables. For instance, in [17, a simple bifurcation of a torus from an isolated orbit in the integrable Hamiltonian $H=\left(p_{x}^{2}+p_{y}^{2}\right) / 2+\left(x^{2}+y^{2}\right) / 2-\lambda y^{3} / 3$ could be described by the normal form

$$
S_{1}(I, \epsilon)=S_{0}-\epsilon I+a I^{2}
$$

with suitably chosen constants $S_{0}$ and $a$ and bifurcation parameter $\epsilon$. The situation there corresponds to one half of that seen in figure 3 a torus B bifurcates from an isolated orbit C. The stationary condition for (3.2) is

$$
\frac{\partial S_{1}}{\partial I}\left(I_{\mathrm{B}}, \epsilon\right)=0, \quad \Rightarrow \quad I_{\mathrm{B}}=\frac{\epsilon}{2 a} .
$$

Since $I$ must be positive definite, the torus B exists only for $\epsilon / a>0$; its stability trace is always $\operatorname{Tr} M_{\mathrm{B}}=+2$ there. The isolated parent orbit arises from $I=0$ as a semiclassical end-point correction (see [17] for details). Its stability trace is given by $\operatorname{Tr} M_{\mathrm{C}}=2-\epsilon^{2}$, so that it is always stable except at the bifurcation point $\epsilon=0$.

In this section, we develop normal forms for some of the bridge-bifurcation scenarios described in the previous section. We start with the integrable model (2.6). Figure 10 shows a Poincaré surface of section obtained for the parameters $\alpha=2.4$ and $\kappa=0$, fixing $y=0$ (and $\dot{y}>0$ ). Since the system is integrable, all fixed points lie on continuous curves which are intersections of rational tori with the $\left(x, p_{x}\right)=(q, p)$ plane, except for the two isolated fixed points along the $x$ axis which belong to the isolated rotating orbits $\mathrm{C}_{ \pm}$. The corresponding bifurcation diagrams are seen in figure 1 where the rational tori correspond to the $\mathrm{B}(1,1)$ bridge orbit. The envelope of the curves in figure 10 corresponds to the boundary of the classically accessible phase space given by $p_{y}=0$.

The phase-space diagram seen in figure 10 is completely analogous to that of a two-dimensional isotropic harmonic oscillator $H=\left(p_{x}^{2}+p_{y}^{2}\right) / 2+\omega^{2}\left(x^{2}+y^{2}\right) / 2$. The tori correspond to the elliptic orbits, the isolated points to the circular orbits $\mathrm{C}_{ \pm}$(with 
both time orientations), and the line $x=0$ to the librating orbits. (The boundary contains the librating orbits along the $y$ axis, i.e., with $p_{y}=0$, which strictly cannot be seen in the Poincaré plot.) It is useful to parameterize the orbits by their (conserved) angular momentum $L=x p_{y}-y p_{x}$. The circular orbits $\mathrm{C}_{+}$and $\mathrm{C}_{-}$have maximum and minimum value of $L$, respectively, and all intermediate nonzero $L$ values correspond to elliptic orbits which are degenerate against rotations around the origin about an angle $\phi \in[0, \pi) . L=0$ corresponds to the librating orbits which have the same degeneracy.

This analogy suggests to use the angular momentum $L$ and the rotation angle $\phi$ of the harmonic-oscillator orbits to define action-angle variables $\varphi=2 \phi$ and $I=L / 2$ and map these onto the Poincaré variables of our present integrable system (2.6). This transformation is derived in Appendix A. The resulting mapping of $(\varphi, I)$ to the Cartesian Poincaré variables $(q, p)$ is given by the relations (here for $\omega=1$ )

$$
\begin{aligned}
q & =\sqrt{2 \rho} \frac{\cos \theta}{\sqrt{1-\sin \theta \cos \varphi}}, & & \\
p & =\sqrt{2 \rho} \frac{\sin \theta \sin \varphi}{\sqrt{1-\sin \theta \cos \varphi}}, & & \rho>0, \quad 0 \leq \varphi \leq 2 \pi, \\
I & =\frac{1}{2} q \sqrt{4 \rho-q^{2}-p^{2}}=\rho \cos \theta, & & 0 \leq \theta \leq \pi,
\end{aligned}
$$

which define an area-conserving canonical transformation $(\varphi, I) \leftrightarrow(q, p)$. Hereby $\theta$ is an angle parameterizing the angular momentum by $L=L_{c} \cos \theta$, where $L_{c}$ is the angular momentum of the $\mathrm{C}_{+}$orbit in the harmonic oscillator. Note that the transformation defined by (3.4) is more complicated than that given in (3.1); in particular, the action variable $I$ here can have both signs. It is limited by the values $\pm \rho$ yielding the fixed points $(q, p)=( \pm \sqrt{2 \rho}, 0)$ which correspond to the two isolated orbits $\mathrm{C}_{ \pm}$at their bifurcation points where the bridge orbit is created or absorbed.

It turns out now that the bridge bifurcations in the integrable system (2.6) can be described by the same normal form function $S_{1}(I, \epsilon)$ as defined in (3.2) above, except that here we have to use the definition of $I$ in (3.4). As shown in Appendix A, $\rho$ equals $L_{c} / 2$ in the harmonic oscillator model. Here, $\rho>0$ is simply a parameter of the normal form that will be determined in section 4 .

The stationary points of $S_{1}(I, \epsilon)$ are most conveniently found in terms of the variable $\theta$. The stationary condition is

$$
\frac{\partial S_{1}}{\partial \theta}=\rho \sin \theta(\epsilon-2 a I)=0 .
$$

The stationary points satisfying $\sin \theta=0$, i.e., $\theta=0$ and $\pi$, correspond to the two isolated circular orbits $\mathrm{C}_{ \pm}$with $I= \pm \rho$. In addition, there is another stationary point satisfying $I_{\mathrm{B}}=\epsilon / 2 a$ as in (3.3) above, corresponding to the B torus. Since $I_{\mathrm{B}}$ must also fulfill the condition $I_{\mathrm{B}}=\rho \cos \theta$, it has real values only for

$$
-2|a| \rho \leq \epsilon \leq 2|a| \rho,
$$

so that the $\mathrm{B}$ torus only exists in the range of $\epsilon$ values between the bifurcation points of the $\mathrm{C}_{ \pm}$orbits. The actions of these periodic orbits are obtained by inserting their stationary values of $I$ into the normal form (3.2); they become

$$
S_{\mathrm{C}_{ \pm}}=S_{1}( \pm \rho, \epsilon)=S_{0} \mp \epsilon \rho+a \rho^{2}, \quad S_{\mathrm{B}}=S_{1}\left(I_{\mathrm{B}}, \epsilon\right)=S_{0}-\frac{\epsilon^{2}}{4 a} .
$$


The traces of their stability matrices can be obtained from the normal form by [15]

$$
\begin{aligned}
& \operatorname{Tr} M=\left(\frac{\partial^{2} \hat{S}}{\partial p \partial q}\right)^{-1}\left[1+\left(\frac{\partial^{2} \hat{S}}{\partial p \partial q}\right)^{2}-\frac{\partial^{2} \hat{S}}{\partial p^{2}} \frac{\partial^{2} \hat{S}}{\partial q^{2}}\right], \\
& \hat{S}(q, p, \epsilon)=S_{1}(q, p, \epsilon)+q p
\end{aligned}
$$

whereby $\hat{S}(q, p, \epsilon)$ is the generating function of the Poincaré map with initial momentum $p$ and final coordinate $q$ in the $(q, p)$ plane at the value $\epsilon$ of the bifurcation parameter. From this we obtain for our periodic orbits

$$
\operatorname{Tr} M_{\mathrm{C}_{ \pm}}=2-(\epsilon \mp 2 a \rho)^{2}, \quad \operatorname{Tr} M_{\mathrm{B}}=2 .
$$

Hence we see that the normal form (3.2) with the mapping (3.4) correctly describes the bifurcation scenario of the bridge orbits found in the model (2.6), as illustrated in figure 1.

In non-integrable systems, the normal form must depend also on the angle variable $\varphi$. Let us consider the following normal form

$$
\begin{aligned}
S_{2}(I, \varphi, \epsilon) & =S_{0}-\epsilon I+a I^{2}+b\left(\rho^{2}-I^{2}\right) \cos ^{2} \varphi \\
& =S_{0}-\epsilon \rho \cos \theta+\rho^{2}\left(a \cos ^{2} \theta+b \sin ^{2} \theta \cos ^{2} \varphi\right),
\end{aligned}
$$

which respects the fact that the $\varphi$-dependent terms in any canonically invariant quantity (such as action, stability) should vanish for $I= \pm \rho$. The stationary phase conditions become

$$
\begin{aligned}
& \frac{\partial S_{2}}{\partial \varphi}=-2 b \rho^{2} \sin ^{2} \theta \sin \varphi \cos \varphi=0, \\
& \frac{\partial S_{2}}{\partial \theta}=\rho \sin \theta\left(\epsilon-2 \rho \cos \theta\left(a-b \cos ^{2} \varphi\right)\right)=0 .
\end{aligned}
$$

The above set of equations have the following solutions:

$$
\begin{aligned}
& \sin \theta=0, \quad \theta_{ \pm}=0, \pi \quad \Leftrightarrow \quad I_{ \pm}= \pm \rho, \\
& \cos \varphi=0, \quad \varphi_{\mathrm{B} 1}=\frac{\pi}{2}, \frac{3 \pi}{2} \quad \Leftrightarrow \quad I_{\mathrm{B} 1}=\rho \cos \theta_{\mathrm{B} 1}=\frac{\epsilon}{2 a}, \\
& \sin \varphi=0, \quad \varphi_{\mathrm{B} 2}=0, \pi \quad \Leftrightarrow \quad I_{\mathrm{B} 2}=\rho \cos \theta_{\mathrm{B} 2}=\frac{\epsilon}{2(a-b)} .
\end{aligned}
$$

The periodic orbits corresponding to the solutions (3.12b) and (3.12c) exist for $-2|a| \rho<\epsilon<2|a| \rho$ and $-2|a-b| \rho<\epsilon<2|a-b| \rho$, respectively. The actions of these periodic orbits are

$$
\begin{aligned}
& S_{ \pm}=S_{0} \mp \epsilon \rho+a \rho^{2}, \\
& S_{\mathrm{B} 1}=S_{0}-\frac{\epsilon^{2}}{4 a}, \\
& S_{\mathrm{B} 2}=S_{0}-\frac{\epsilon^{2}}{4(a-b)}+b \rho^{2},
\end{aligned}
$$

and the traces of their stability matrices are

$$
\begin{aligned}
\operatorname{Tr} M_{ \pm} & =2-(\epsilon \mp 2 a \rho)[\epsilon \mp 2(a-b) \rho], \\
\operatorname{Tr} M_{\mathrm{B} 1} & =2+\frac{b}{a}(\epsilon-2 a \rho)(\epsilon+2 a \rho), \\
\operatorname{Tr} M_{\mathrm{B} 2} & =2-\frac{b}{a-b}[\epsilon-2(a-b) \rho][\epsilon+2(a-b) \rho] .
\end{aligned}
$$


Thus, the periodic orbits (3.12b) and (3.12c) are the two bridge orbits which connect the two periodic orbits corresponding to (3.12a) at the bifurcation points $\epsilon_{\text {bif }}= \pm 2 a \rho$ and $\pm 2(a-b) \rho$. The bridge orbits found in figure 8 can be regarded as of this type. For $b=a$ (or $a=0$ ) the bridge orbit B2 (or B1) shrinks to a single point $\epsilon=0$. This corresponds to the situation found for the $(1,1)$ orbit in figure 6. The orbit Q in figure 9 can be regarded as the bridge orbit B2 in the limit of extremely small $|a-b|$.

Figure 11] shows the Poincare surface of section for the Hamiltonian (2.12), with deformations given by (2.14), for $\alpha=2.2, \beta_{4}=0.002$ and several values of $\beta$ along the right half of the $(1,1)$ bridge bifurcation region (cf. the left panel of figure 8 ). The Poincaré variables $(q, p)$ are here defined by $q=(x+y) / \sqrt{2}, p=\left(p_{x}+p_{y}\right) / \sqrt{2}$ at the surface of section $x-y=0, \dot{x}-\dot{y}>0$. At $\beta=0$, the existing periodic orbits are the bridge $\mathrm{B} 1$ at $(q, p) \approx(0, \pm 1)$, the bridge $\mathrm{B} 2$ at $(q, p)=(0,0)$, and the diameter orbits $\mathrm{A}_{x}, \mathrm{~A}_{y}$ at $(q, p) \approx(\mp 1,0)$. Both bridges are stable and the diameters are unstable. At the bifurcation point $\beta=0.016$, the bridge $\mathrm{B} 2$ merges into the $\mathrm{A}_{x}$ orbit and $\mathrm{A}_{x}$ becomes stable. At the bifurcation point $\beta=0.077$, the bridge $\mathrm{B} 1$ merges into the $\mathrm{A}_{y}$ orbit and $\mathrm{A}_{y}$ becomes stable. By fitting (3.13) and (3.14) to the periodic orbit quantities for $\alpha=2.2$ and $\beta_{4}=0.002$, we obtain $a \rho=-0.12$ and $b \rho=-0.145$. The bifurcation points are thus $\epsilon= \pm 0.24$ and \pm 0.05 for the B1 and B2 bridges, respectively. Figure 12 shows the contour map of the normal form (3.10) with the above values of $a$ and $b$, and with $\epsilon$ corresponding to each value of $\beta$ in figure 11, One will see that the phase space profiles of figure 11 are nicely reproduced by this normal form.

For the asymmetric bridges $\left(n_{+}, n_{-}\right)$with $n_{+} \neq n_{-}$we have so far not found any suitable normal form. One may need a mapping based on the anisotropic (rational) harmonic oscillator instead of that given in (3.4) which is based on the isotropic oscillator. This will be investigated in further research.

\section{Uniform approximations for the density of states}

\subsection{Local uniform approximation}

In this section we evaluate the semiclassical level density around the bifurcation points using the normal form obtained in the above section. In the following we limit ourselves to the integrable model described by the Hamiltonian (2.6). The calculation of the parameters in a normal form from a given Hamiltonian is in general a very difficult problem. The idea of the uniform approximations is to avoid their direct calculation by relating them to the local invariant properties of the participating periodic orbits in the vicinity of a bifurcation, which can be obtained numerically from a periodicorbit search. The bridge bifurcations occurring in the Hamiltonian (2.6) involve three orbits: the two circular orbits $\mathrm{C}_{ \pm}$(with repetition numbers $n_{+}$and $n_{-}$) and the bridge orbits $\mathrm{B}\left(n_{+}, n_{-}\right)$(cf. figures 1, 3). From these, we can determine five independent quantities: the three actions $S_{ \pm}$and $S_{\mathrm{B}}$, and the two stability traces $\operatorname{Tr} M_{ \pm}$(recall that $\operatorname{Tr} M_{\mathrm{B}}=2$ is constant). The normal form given by (3.2) and (3.4) contains the four parameters $S_{0}, \epsilon, a$ and $\rho$, which we can determine using four of the above five orbit properties. In problems with only one bifurcation point, such a way of determining the normal form parameters does not contradict with remaining unused quantities, but in the present situation of the bridge bifurcations, the values of the parameters do depend on which quantities are used. This problem is related with the global nature of the bridge bifurcations, where the parameters undergo significant changes 

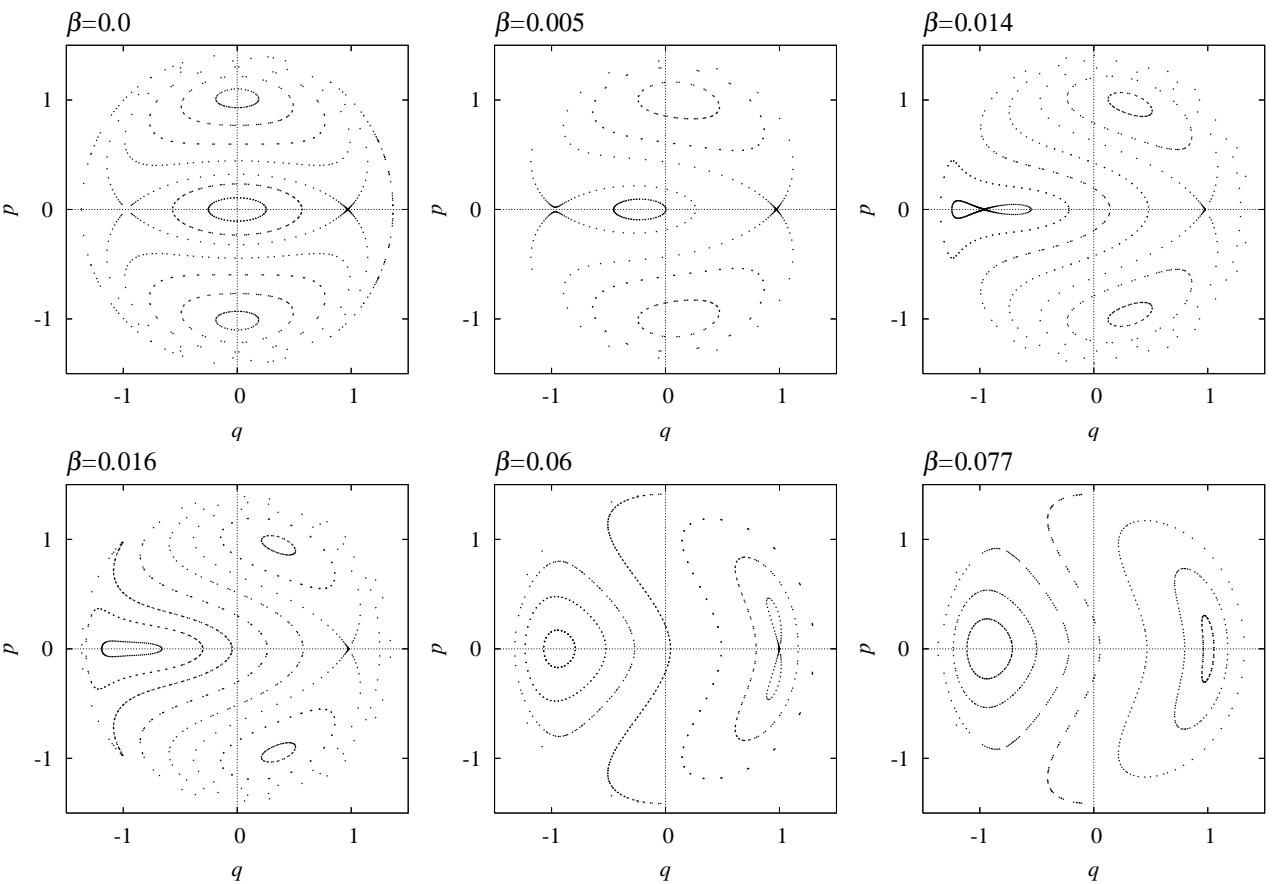

Figure 11. Poincaré surface of section for the Hamiltonian (2.12) with deformation (2.14), plotted for $\alpha=2.2, \beta_{4}=0.002$ and several values of $\beta$ in the $(1,1)$ bifurcation region. (See text for definitions of the Poincaré variables $(q, p)$.)
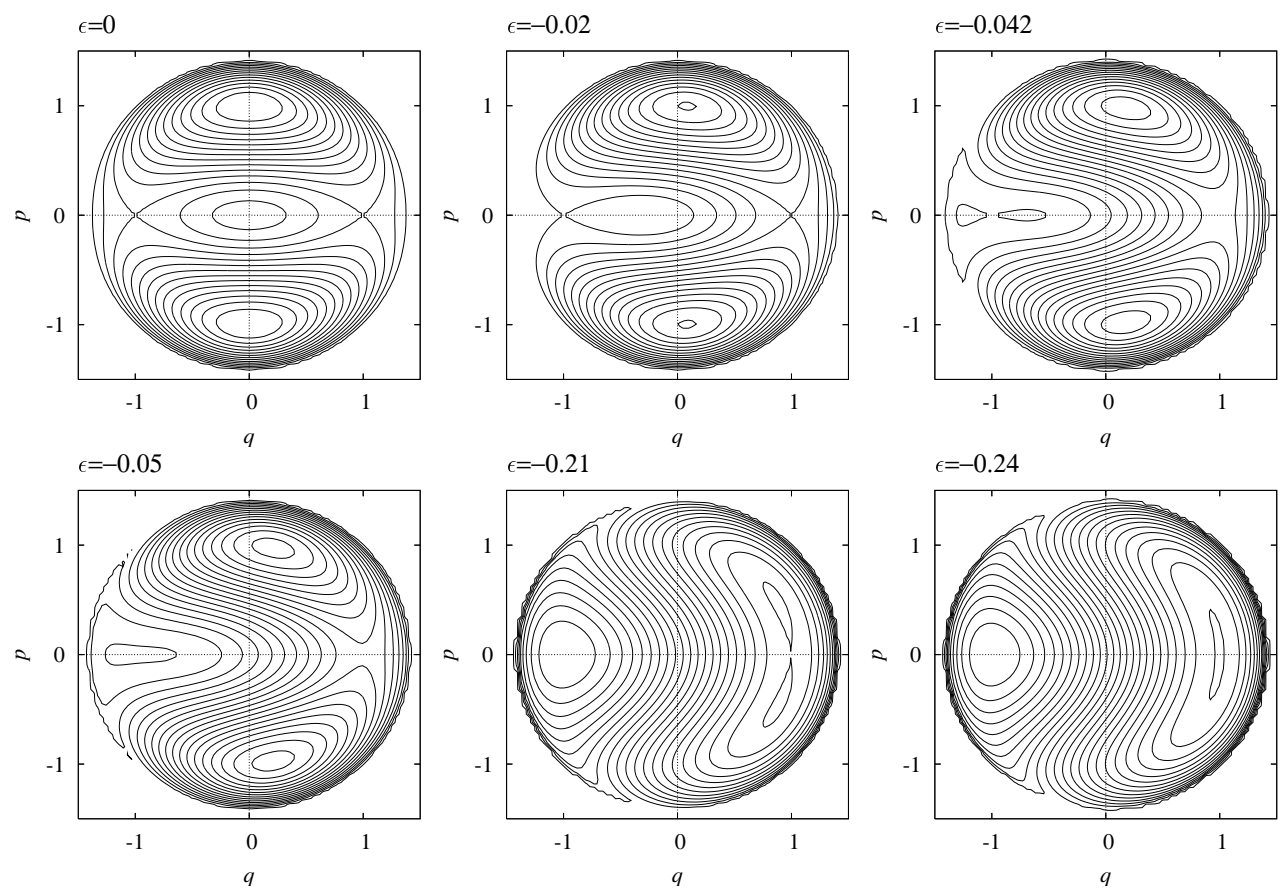

Figure 12. Contour map of the normal form (3.10) with $a \rho=-0.12, b \rho=-0.145$ and several values of $\epsilon$ in bifurcation region corresponding to each panels of figure $11(q, p)$ are plotted in units of $\sqrt{2 \rho}$. 
between the two bifurcation points. Different from all bifurcations treated so far in the literature in uniform approximations, the bridge orbit here has no 'external link', i.e., there is no external orbit outside the bifurcation interval to which its properties can be asymptotically linked. This leads to a slight ambiguity in determining the parameter $a$, as we shall see below.

In order to determine the normal form parameters uniquely, at least locally for each given $\epsilon$, we add one more term to $S_{1}(I, \epsilon)$ in (3.2) and use the normal form

$$
S_{3}(I, \epsilon)=S_{0}+\epsilon I+a I^{2}+b I^{3}, \quad I=\rho \sin \theta .
$$

The stationary-phase analysis then predicts the properties of the periodic orbits to be

$$
\begin{aligned}
& S_{ \pm}=S_{0}+a \rho^{2} \mp\left(\epsilon \rho-b \rho^{3}\right) \\
& S_{\mathrm{B}}=S_{0}-\frac{\epsilon^{2}\left(1+2 b \epsilon / a^{2}+\sqrt{1+3 b \epsilon / a^{2}}\right)}{a\left(1+\sqrt{1+3 b \epsilon / a^{2}}\right)^{3}} \\
& \operatorname{Tr} M_{ \pm}=2-\left[\epsilon-\left( \pm 2 a \rho+3 b \rho^{2}\right)\right]^{2} .
\end{aligned}
$$

The five normal form parameters $\left(S_{0}, \epsilon, a, b\right.$, and $\left.\rho\right)$ are now uniquely determined by the five equations in (4.2), although these cannot be solved analytically. Due to the scaling rules, the parameters have the following energy dependences:

$$
S_{0}=\hbar \mathcal{E} \tau_{0}, \quad \rho=\hbar \mathcal{E} \tilde{\rho}, \quad a=\frac{\tilde{a}}{\hbar \mathcal{E}}, \quad b=\frac{\tilde{b}}{(\hbar \mathcal{E})^{2}},
$$

where $\tau_{0}, \tilde{\rho}, \tilde{a}$, and $\tilde{b}$ are dimensionless constants. Ideally, these four parameters should not depend on the bifurcation parameter $\epsilon$ throughout the bifurcation region.

Figure 13 shows their results which we have determined numerically for the $(1,1)$ and $(2,1)$ bridge-orbit bifurcations for $\alpha=2.02$. In the center panels, we show besides $\tau_{0}$ also the scaled periods $\tau$ of all three periodic orbits. The parameters $a, b, S_{0}$ and $\rho$ turn out to be approximately constant throughout the bifurcation region, as hoped, in particular for the symmetric $(1,1)$ bridge. Note that at $\epsilon=0$, the value of $b$ is exactly zero for the symmetric bridge bifurcation, so that the cubic term in (4.1) does not contribute there. Furthermore, the contribution of the cubic term $b I^{3}$ to the actions of the periodic orbits remains much smaller than that of the quadratic term $a I^{2}$ throughout the whole bifurcation region in both cases $(1,1)$ and $(2,1)$. It will therefore be omitted again in the following.

The combined contribution of all orbits involved in the bifurcation to the semiclassical level density is given by 9,10

$\delta g(E)=\frac{1}{2 \pi^{2} \hbar^{2}} \operatorname{Re} \int \mathrm{d} q \int \mathrm{d} p \Psi(q, p, \epsilon) \exp \left[\frac{\mathrm{i}}{\hbar}\{\hat{S}(q, p, \epsilon)-q p\}-\frac{\mathrm{i} \pi}{2} \nu\right]$,

where $\hat{S}(q, p, \epsilon)$ is the generating function defined in (3.8) which contains the appropriate normal form, and the amplitude function $\Psi(q, p, \epsilon)$ is given by

$$
\Psi(q, p, \epsilon)=\frac{\partial \hat{S}}{\partial E}\left|\frac{\partial^{2} \hat{S}}{\partial q \partial p}\right|^{1 / 2} .
$$

Here $\nu$ is the Maslov index of the bridge orbit labeled by $\left(n_{+}, n_{-}\right)$. It is given by

$$
\nu=2 n_{r}, \quad n_{r}=n_{+}+n_{-},
$$

where $n_{r}$ is equal to the number of librations in the radial direction. The amplitude function $\Psi(q, p, \epsilon)$ usually has a moderate dependence on the variables $q, p, \epsilon$ and can 

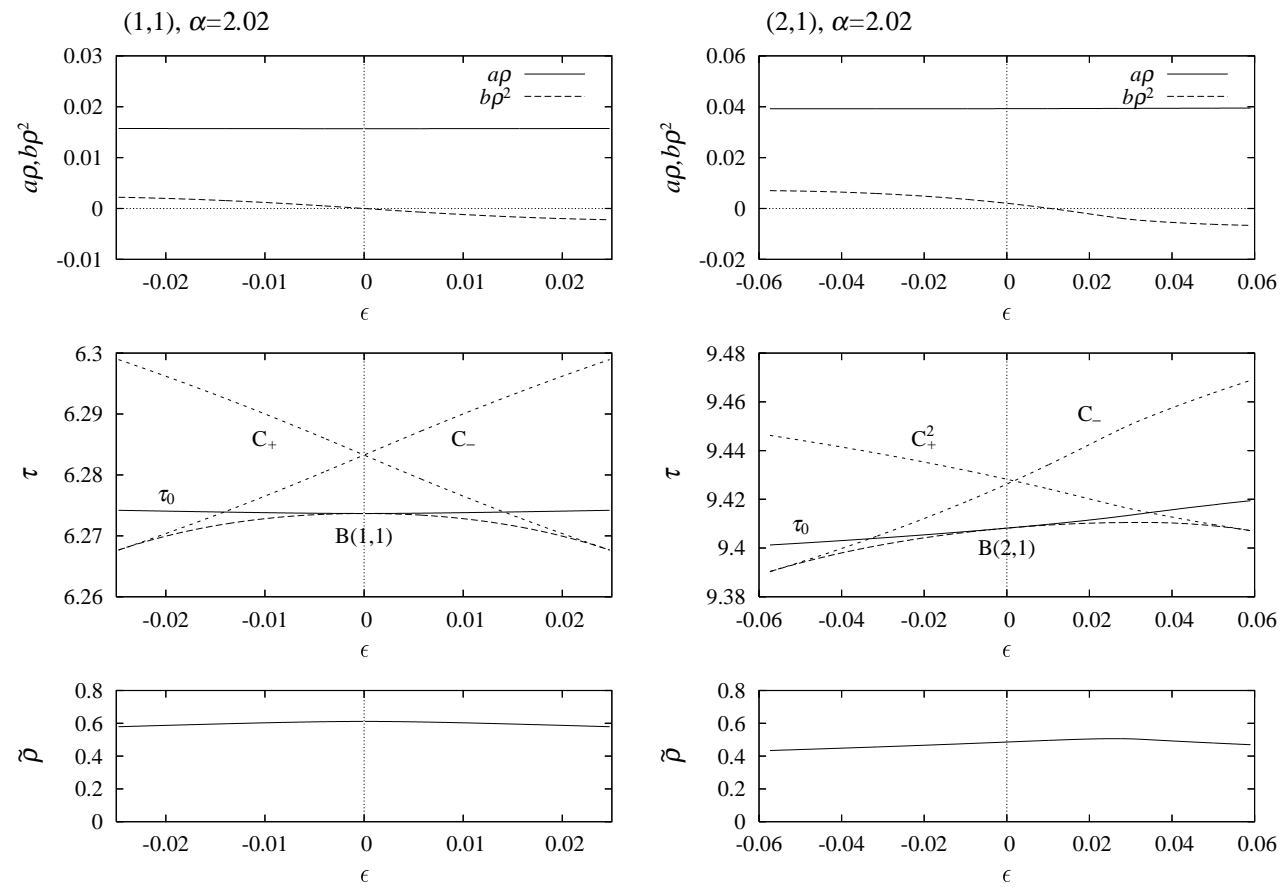

Figure 13. Normal form parameters determined from periodic orbit quantities. Left and right panels are results for the $(1,1)$ and $(2,1)$ bridge bifurcations, respectively, for $\alpha=2.02$. In the center panels, the scaled periods $\tau$ of all three orbits are shown besides $\tau_{0}=S_{0} / \hbar \mathcal{E}$.

be replaced by its value at the origin, $\Psi(0,0,0)=\partial S_{0} / \partial E=T_{0}$, which is the average period of the orbit cluster. By transforming the variables from $(q, p)$ to $(\varphi, I)$ and integrating over $\varphi$, one obtains

$$
\delta g(E) \simeq \frac{T_{0}}{\pi \hbar^{2}} \operatorname{Re} \mathrm{e}^{\mathrm{i}\left(S_{0} / \hbar-\pi \nu / 2\right)} \int_{-\rho}^{\rho} \mathrm{d} I \mathrm{e}^{\mathrm{i}\left(-\epsilon I+a I^{2}\right) / \hbar} .
$$

Here, we omitted the cubic term $b I^{3}$ in the normal form (4.1) as stated above. The integral on the right-hand side can be analytically expressed as

$$
\begin{aligned}
& \int_{-\rho}^{\rho} \mathrm{d} I \mathrm{e}^{\mathrm{i}\left(-\epsilon I+a I^{2}\right) / \hbar}=\sqrt{\frac{2 \pi \hbar}{4 a}} \mathrm{e}^{\mathrm{i} \epsilon^{2} / 4 a \hbar}\left\{\sigma_{+}\left(c_{+}+\mathrm{i} s_{+}\right)+\sigma_{-}\left(c_{-}+\mathrm{i} s_{-}\right)\right\}, \\
& c_{ \pm}=\mathrm{C}\left(\left|x_{ \pm}\right|\right), \quad s_{ \pm}=\mathrm{S}\left(\left|x_{ \pm}\right|\right), \quad x_{ \pm}=\sqrt{\frac{4 a}{2 \pi \hbar}}\left(\rho \mp \frac{\epsilon}{2 a}\right), \quad \sigma_{ \pm}=\operatorname{sgn} x_{ \pm},
\end{aligned}
$$

where $\mathrm{C}(x)$ and $\mathrm{S}(x)$ are the Fresnel functions defined by

$$
\mathrm{S}(x)=\int_{0}^{x} \sin \left(\frac{\pi}{2} t^{2}\right) \mathrm{d} t, \quad \mathrm{C}(x)=\int_{0}^{x} \cos \left(\frac{\pi}{2} t^{2}\right) \mathrm{d} t .
$$

Inserting (4.8) into (4.7), one obtains the local uniform approximation to the level density:

$\delta g(E)=\frac{T_{0}}{\hbar^{3 / 2} \sqrt{2 \pi a}} \operatorname{Re} \mathrm{e}^{\mathrm{i}\left(S_{\mathrm{B}} / \hbar-\pi \nu / 2\right)}\left\{\sigma_{+}\left(c_{+}+\mathrm{i} s_{+}\right)+\sigma_{-}\left(c_{-}+\mathrm{i} s_{-}\right)\right\}$. 
In terms of the scaled dimensionless normal form parameters given in (4.3), the scaled level density becomes

$\delta g(\mathcal{E})=\sqrt{\frac{\mathcal{E}}{2 \pi \tilde{a}}} \tau_{0} \operatorname{Re} \mathrm{e}^{\mathrm{i}\left(\tau_{B} \mathcal{E}-\pi \nu / 2\right)}\left\{\sigma_{+}\left(c_{+}+\mathrm{i} s_{+}\right)+\sigma_{-}\left(c_{-}+\mathrm{i} s_{-}\right)\right\}$,

where $\tau_{\mathrm{B}}=S_{\mathrm{B}} / \hbar \mathcal{E}=\tau_{0}+\tilde{\epsilon}^{2} / 4 \tilde{a}$ is the scaled period of the bridge orbit, $c_{ \pm}$and $s_{ \pm}$ are given by (4.9) with the arguments

$$
x_{ \pm}=\sqrt{\frac{2 \tilde{a} \mathcal{E}}{\pi}}\left(\tilde{\rho} \mp \frac{\epsilon}{2 \tilde{a}}\right) .
$$

\subsection{Global uniform approximation}

The local uniform approximation (4.11) is valid only in the vicinities of the bifurcation points, where the properties of periodic orbits are nicely described by the normal form (3.2). Far from the bifurcation points in the $\epsilon$ variable, the standard asymptotic trace formulae should work, which are given in the form of the Gutzwiller formula 1 for the isolated orbits and the Berry-Tabor formula [30] for the family of bridge orbits. The purpose of the so-called global uniform approximations is to interpolate between the local result (4.11) and the standard trace formulae. For the generic bifurcations, such global uniform approximations were developed by Sieber and Schomerus [13, 14, 15. We shall presently follow their procedure to derive a global uniform approximation for the integrable bridge bifurcations.

In order to go beyond the local uniform approximation, one can start from equation (4.4), but one has to expand the amplitude function $\Psi(q, p, \epsilon)$ around the bifurcation point in a way similar to the normal form function $S(q, p, \epsilon)$ for the action integral. We find that it is sufficient here to take into account only a linear term in $I$. We thus write $\delta g(E)$ as

$\delta g(E)=\frac{1}{\pi \hbar^{2}} \operatorname{Re} \int_{-\rho}^{\rho} \mathrm{d} I(\alpha+\beta I) \exp \left[\frac{\mathrm{i}}{\hbar}\{\hat{S}(q, p)-q p\}-\frac{\mathrm{i} \pi}{2} \nu\right]$.

In principle, the parameters $\alpha$ and $\beta$ are determined from higher-order expansion coefficients in the normal form (cf. [14]), but in practice one may determine them from the condition that the integral (4.14) reproduces the asymptotic contributions of the bifurcating orbits to the standard trace formulae far from the bifurcation points. There, the action differences between different periodic orbits corresponding to the stationary points of the normal form (3.2) are much larger than $\hbar$. This is equivalent to taking the asymptotic expansions of the Fresnel integrals when their arguments are much larger than unity,

$$
x_{ \pm}=\sqrt{\frac{2\left(a \rho^{2} \mp \epsilon \rho+\epsilon^{2} / 4 a\right)}{\pi \hbar}}=\sqrt{\frac{2}{\pi} \frac{S_{ \pm}-S_{\mathrm{B}}}{\hbar}} \gg 1 .
$$

Their asymptotic forms are (see e.g., 31])

$$
\begin{aligned}
& \mathrm{C}(x) \simeq \frac{1}{2}+\frac{1}{\pi x} \sin \frac{\pi}{2} x^{2}, \quad \mathrm{~S}(x) \simeq \frac{1}{2}-\frac{1}{\pi x} \cos \frac{\pi}{2} x^{2}, \\
& \mathrm{C}(x)+\mathrm{i} \mathrm{S}(x) \simeq \frac{\mathrm{e}^{\mathrm{i} \pi / 4}}{\sqrt{2}}-\frac{\mathrm{i}}{\pi x} \mathrm{e}^{\mathrm{i} \pi x^{2} / 2}, \quad x \gg 1 .
\end{aligned}
$$


Their contribution to the level density is then given by

$$
\begin{aligned}
\delta g(E)=\frac{1}{\pi \hbar^{2}} \operatorname{Re} \mathrm{e}^{\mathrm{i}\left(S_{0} / \hbar-\pi \nu / 2\right)} \int_{-\rho}^{\rho} \mathrm{d} I(\alpha+\beta I) \mathrm{e}^{\mathrm{i}\left(-\epsilon I+a I^{2}\right)} \\
=\frac{1}{\pi \hbar^{2}} \operatorname{Re}\left[\sqrt{\frac{2 \pi \hbar}{4 a}}\left(\alpha+\frac{\beta \epsilon}{2 a}\right) \mathrm{e}^{\mathrm{i}\left(S_{\mathrm{B}} / \hbar-\pi \nu / 2\right)}\left\{\sigma_{+}\left(c_{+}+\mathrm{i} s_{+}\right)+\sigma_{-}\left(c_{-}+\mathrm{i} s_{-}\right)\right\}\right. \\
\left.+\frac{\beta \hbar}{2 a}\left(\mathrm{e}^{\mathrm{i}\left(S_{+} / \hbar-\pi(\nu+1) / 2\right)}-\mathrm{e}^{\mathrm{i}\left(S_{-} / \hbar-\pi(\nu+1) / 2\right)}\right)\right]
\end{aligned}
$$

with $S_{\mathrm{B}}$ and $S_{ \pm}$given by (3.7). Inserting (4.15), one has

$$
\begin{aligned}
\delta g(E) \simeq & \frac{\alpha+\frac{\epsilon \beta}{2 a}}{\pi \hbar^{2} \sqrt{\pi a / \hbar}}\left(\frac{\sigma_{+}+\sigma_{-}}{2}\right) \cos \left(\frac{S_{\mathrm{B}}}{\hbar}-\frac{\pi}{2} \nu+\frac{\pi}{4}\right) \\
& +\frac{\alpha+\beta \rho}{\pi \hbar|2 a \rho-\epsilon|} \cos \left(\frac{S_{+}}{\hbar}-\frac{\pi}{2}\left(\nu+\sigma_{+}\right)\right) \\
& +\frac{\alpha-\beta \rho}{\pi \hbar|2 a \rho+\epsilon|} \cos \left(\frac{S_{-}}{\hbar}-\frac{\pi}{2}\left(\nu+\sigma_{-}\right)\right) .
\end{aligned}
$$

The first term on the right-hand side can be identified as the contribution of the bridge orbit with its Berry-Tabor amplitude

$$
A_{\mathrm{BT}}=\frac{T_{0}}{\pi \hbar^{2}} \sqrt{\frac{2 \pi \hbar}{|\mathcal{K}|}}, \quad \mathcal{K}=\frac{\partial^{2} S}{\partial I^{2}}
$$

and we have

$$
\alpha+\frac{\epsilon \beta}{2 a}=T_{\mathrm{B}}, \quad a=\frac{1}{2} \mathcal{K},
$$

$T_{\mathrm{B}}$ being the period of the primitive bridge orbit. The second and third terms in the right-hand side of (4.17) are identified as the contributions of two isolated orbits with their Gutzwiller amplitudes

$$
A_{ \pm}=\frac{T_{ \pm}}{\pi \hbar n_{ \pm} \sqrt{\left|2-\operatorname{Tr} M_{ \pm}\right|}}
$$

where $T_{ \pm}$are the full periods of these orbits and $n_{ \pm}$are their repetition numbers. Furthermore one finds

$$
\alpha \pm \beta \rho=T_{ \pm}, \quad 2 a \rho \pm \epsilon=\sigma_{ \pm} n_{ \pm} \sqrt{\left|2-\operatorname{Tr} M_{ \pm}\right|} .
$$

From (4.20) and (4.21), we can determine the parameters $\alpha$ and $\beta$ by

$$
\alpha=\frac{1}{2}\left(T_{+}+T_{-}\right), \quad \frac{\beta}{2 \pi \hbar a}=\frac{T_{+}-T_{-}}{\frac{\sigma_{+} T_{+}}{A_{+}}+\frac{\sigma_{-} T_{-}}{A_{-}}} .
$$

Inserting them into (4.16), we obtain the global uniform approximation for the level density

$$
\begin{aligned}
\delta g(E)= & \frac{A_{\mathrm{BT}}}{\sqrt{2}} \operatorname{Re}\left[\mathrm{e}^{\mathrm{i}\left(S_{\mathrm{B}} / \hbar-\pi \nu / 2\right)}\left\{\sigma_{+}\left(c_{+}+\mathrm{i} s_{+}\right)+\sigma_{-}\left(c_{-}+\mathrm{i} s_{-}\right)\right\}\right] \\
& +\frac{T_{+}-T_{-}}{\frac{\sigma_{+} T_{+}}{A_{+}}+\frac{\sigma_{-} T_{-}}{A_{-}}}\left\{\cos \left(\frac{S_{+}}{\hbar}-\frac{\pi}{2}(\nu+1)\right)-\cos \left(\frac{S_{-}}{\hbar}-\frac{\pi}{2}(\nu+1)\right)\right\}
\end{aligned}
$$


with the arguments of the Fresnel integrals given by

$$
x_{ \pm}=\sqrt{\frac{2}{\pi} \frac{S_{ \pm}-S_{\mathrm{B}}}{\hbar}}
$$

Note that all quantities entering this formula are determined from the invariant properties of the periodic orbits.

The global uniform approximation (4.23) is the main result of this section. We shall refer to it in the following as UA2. It becomes important in particular when the two bifurcations, at which the bridge orbits are created and annihilated, are close to each other so that neither of the isolated circular orbits are in the asymptotic region; the two bifurcations then cannot be treated separately. Sufficiently far from the bifurcation points, where all orbits reach their asymptotic domains, one can use the asymptotic trace formulae, referred to as ASY in the following, i.e., the Gutzwiller formula [1] for the isolated circular orbits and the Berry-Tabor formula [30] for the bridge orbit families. If this is also the case for a central region between the two bifurcations, where the bridges are present, their bifurcations from/into the isolated orbits can be treated separately in the uniform approximation given in [17, which we shall refer to as UA1.

\subsection{Numerical results}

In the following, we test numerically the various semiclassical approximations to the level density. We restrict ourselves to the coarse-grained level density in the scaled energy variable $\mathcal{E}$, which quantum-mechanically is defined in terms of the scaled energy spectrum $\left\{\mathcal{E}_{i}\right\}$ by a sum over normalized Gaussians with width $\gamma$ :

$$
g_{\gamma}(\mathcal{E})=\frac{1}{\sqrt{\pi} \gamma} \sum_{i} \exp \left\{-\left(\mathcal{E}-\mathcal{E}_{i}\right)^{2} / \gamma^{2}\right\}
$$

In the semiclassical trace formulae, each contribution of a periodic orbit (po) then has to be multiplied by an exponential damping factor $\exp \left\{-\left(\gamma \tau_{\mathrm{po}} / 2\right)^{2}\right\}$ (see e.g., [7]), so that the contributions of longer orbits are suppressed and the sum over the periodic orbits converges. In our numerical results given below, we have used $\gamma=0.3$ in all cases. With this value of $\gamma$, the condition for the above damping factor to be smaller than $10^{-2}$ corresponds to $\tau_{\text {po }}>14$, whose contributions can be safely neglected.

In figure 14 the oscillating part of the scaled level densities $\delta g(\mathcal{E})$, labeled by ASY and UA1, are compared with the quantum-mechanical result (QM) for the system (2.6) with $\alpha=2.4$ and $\kappa=0.31$. These values of the parameters correspond to a point close to the left bifurcation of the bridge orbit $\mathrm{B}(2,1)$. In calculating the semiclassical level densities, we take into account the circular periodic orbits $\mathrm{C}_{+}^{n_{+}}$with repetition numbers $n_{+} \leq 3$, the orbit $C_{-}$and the bridge family $\mathrm{B}(2,1)$ (see figure 1 for the values of their scaled periods). We see that the uniform approximation UA1 for the bifurcating circular orbits with the bridge orbit improves the semiclassical level density over the asymptotic one and nicely reproduces the quantum results near the bifurcation points.

Figure 15 shows the result of the global uniform approximation UA2 for $\alpha=2.02$. $\kappa=0.0025$ and $\kappa=0.322$ correspond to the bifurcation points of the bridge orbits $\mathrm{B}(1,1)$ and $\mathrm{B}(2,1)$, respectively. In the periodic orbit sum, all periodic orbits with $\tau<15$ are included; namely, $\mathrm{C}_{ \pm}^{n}$ and bridge $\mathrm{B}(n, n)$ with $n \leq 3$ for $\kappa=0.0025$, and $\mathrm{C}_{+}^{n_{+}}$with $n_{+} \leq 3, \mathrm{C}_{-}$and $\mathrm{B}(2,1)$ for $\kappa=0.322$. In comparison to the separate uniform treatment of the two bifurcations (approximation UA1), the UA2 formula 


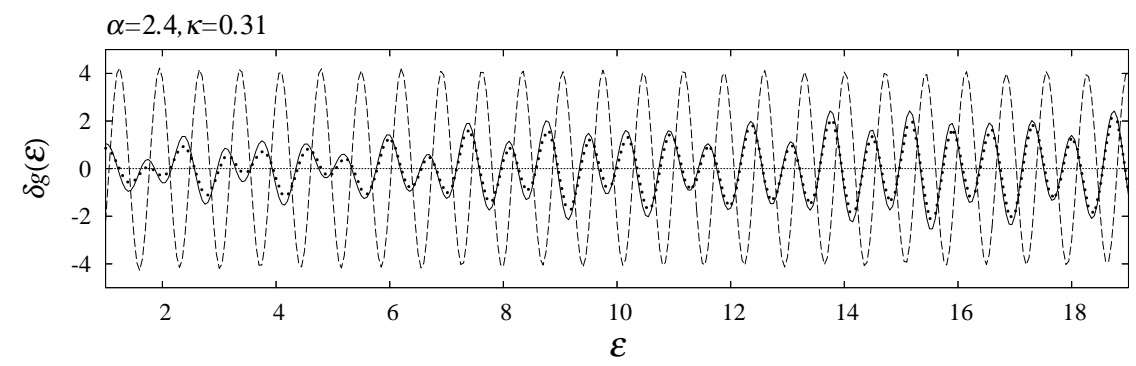

Figure 14. Oscillating part of the scaled level density $\delta g(\mathcal{E})$ for the Hamiltonian (2.6) with $\alpha=2.4$ and $\kappa=0.31$. Solid, dashed and dotted curves represent UA1, ASY (divided by 10) and QM, respectively. In the semiclassical level densities, the periodic orbit sum includes the $\mathrm{C}_{+}^{n_{+}}$orbits with $n_{+} \leq 3$, the orbit $\mathrm{C}_{-}$, and the bridge $\mathrm{B}(2,1)$.
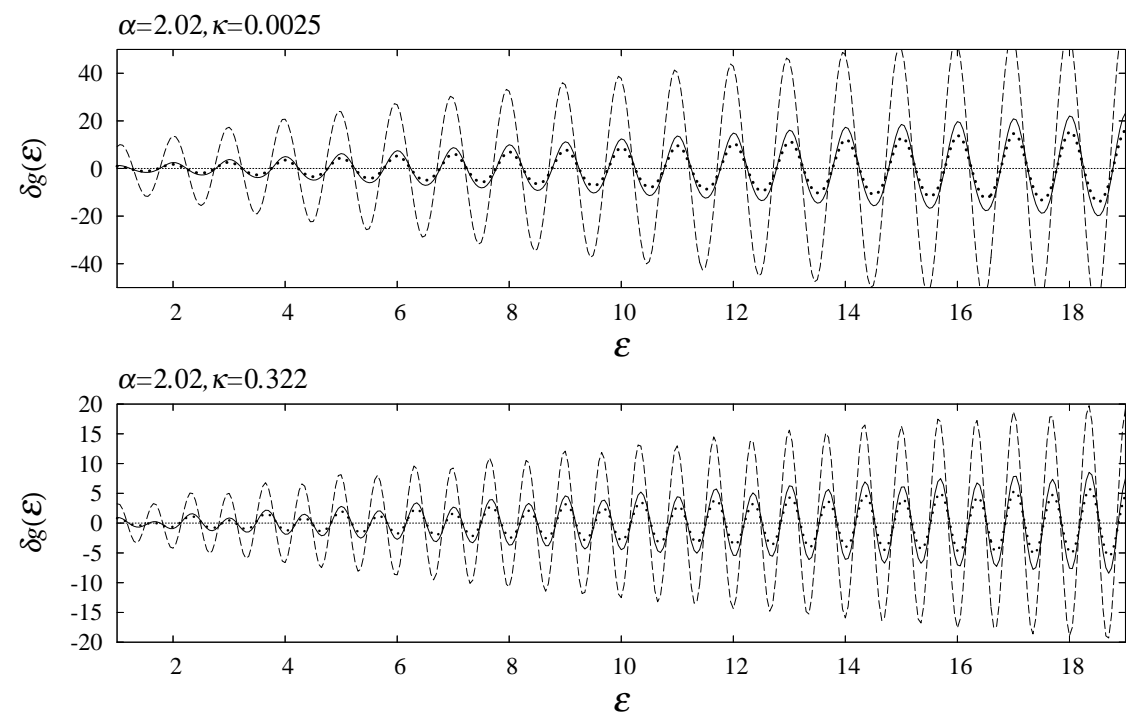

Figure 15. Comparison of uniform approximations for $\alpha=2.02$. Solid, dashed and dotted curves represent UA2, UA1 and QM, respectively. Upper and lower panels are calculated at the bifurcation points of symmetric bridge $(1,1)$ and asymmetric bridge $(2,1)$, respectively. In the upper panel, the periodic orbit sum in the semiclassical level density is taken over $\mathrm{C}_{ \pm}^{n_{ \pm}}$and the bridge $\mathrm{B}\left(n_{+}, n_{-}\right)$with $n_{ \pm} \leq 3$, while in the lower panel, $\mathrm{C}_{+}^{n_{+}}$with $n_{+} \leq 3, \mathrm{C}_{-}$and the bridge $\mathrm{B}(2,1)$ are taken into account.

(4.17) reasonably improves the level density, but we obtain a slight overestimation. Let us consider the origin of this deviation. We note that for $\alpha \sim 2$, there is no asymptotic region between the two bifurcation points. Therefore, the procedure to determine the parameters to reproduce the Berry-Tabor asymptotic form is not justified. In the UA2, the normal form parameter $a$ is determined such that the asymptotic form reproduces the Berry-Tabor trace formula. This corresponds to using the curvature $\mathcal{K}$ of the torus for the normal form parameter $a$ as in (4.19). Figure 16 compares the values of $a$ determined by the two methods, i.e., by (4.19) and (4.2). They are 

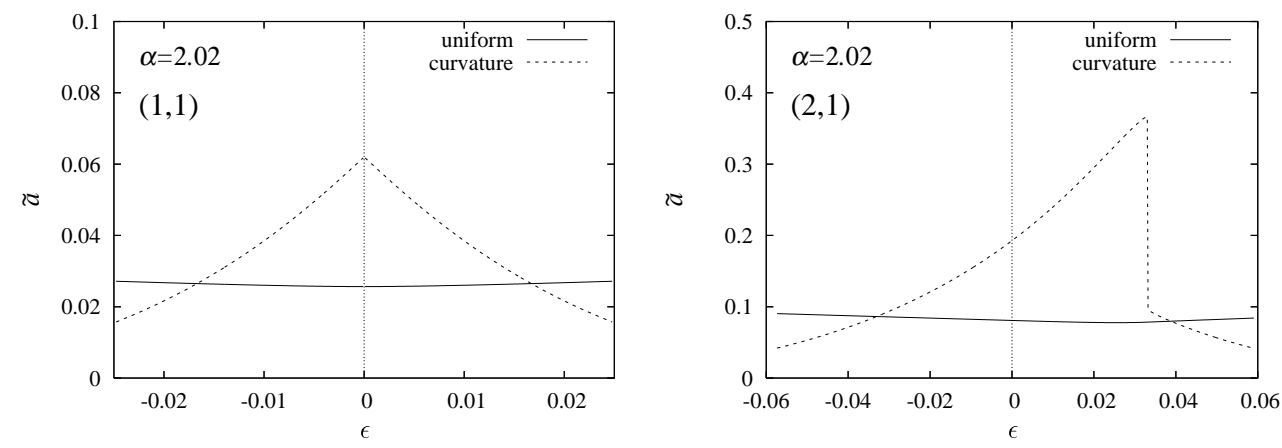

Figure 16. Normal form parameter $\tilde{a}=\hbar \mathcal{E} a$ (see 4.3) determined from 4.2 (solid curve) and that from (4.19) (dashed curve). Left and right panels show the results for $(1,1)$ and $(2,1)$ bridge orbits, respectively.

significantly different from each other, and the global uniform approximation (UA2) does not coincide with the local uniform approximation (4.11) in the bifurcation region.

Using the local uniform approximation (4.11) with the normal form parameters given by (4.2) (referred to as UA2L), the results are much improved near the bifurcation points as shown in figure 17, but at the middle of the bridge, the quantum results lie between $\mathrm{UA} 2$ and $\mathrm{UA2L}$.

Our numerical results can be interpreted as follows. The normal form (3.2) or (4.1) is constructed to reproduce the bifurcation properties of the three participating periodic orbits. Therefore, it is most reliable around the bifurcation points $\epsilon \sim \epsilon_{\text {bif }}$. However, the bridge orbit undergoes large changes between the two bifurcation points which are globally separated in the phase space, and therefore higher-order terms in $I$ in the normal form could contribute significantly in the middle region of the bridge. In fact, the curvature $\mathcal{K}$ of the torus, which is an invariant property of the periodic orbit family and important for determining its contribution to the level density, is not correctly described by the form (4.1), as shown in figure 16. When the parameter $a$ is shifted toward the value determined by the curvature $\mathcal{K}$, the agreement between the local uniform approximation and the quantum results becomes better. This could be achieved by normal forms with higher-order terms in $I$ which, however, would render the global uniform approximation more complicated and less analytic.

\section{Summary}

We have investigated the appearance of bridge orbits, which connect two isolated orbits via two successive bifurcations near the points where their periods and stabilities coincide, in various Hamiltonian systems with two degrees of freedom. In a class of integrable systems, the bridge orbits form degenerate families. For these we used a mapping derived from the Poincaré variables of the isotropic harmonic oscillator to derive a very simple normal form from which all the invariant properties of the participating periodic orbits can be derived analytically. Using this normal form, we have derived analytical uniform approximations for the semiclassical level density of the corresponding quantum systems. Although the normal form parameters could not be determined uniquely and their values undergo slight variations between the two bifurcations, the numerical agreement between the semiclassical and the quantum- 

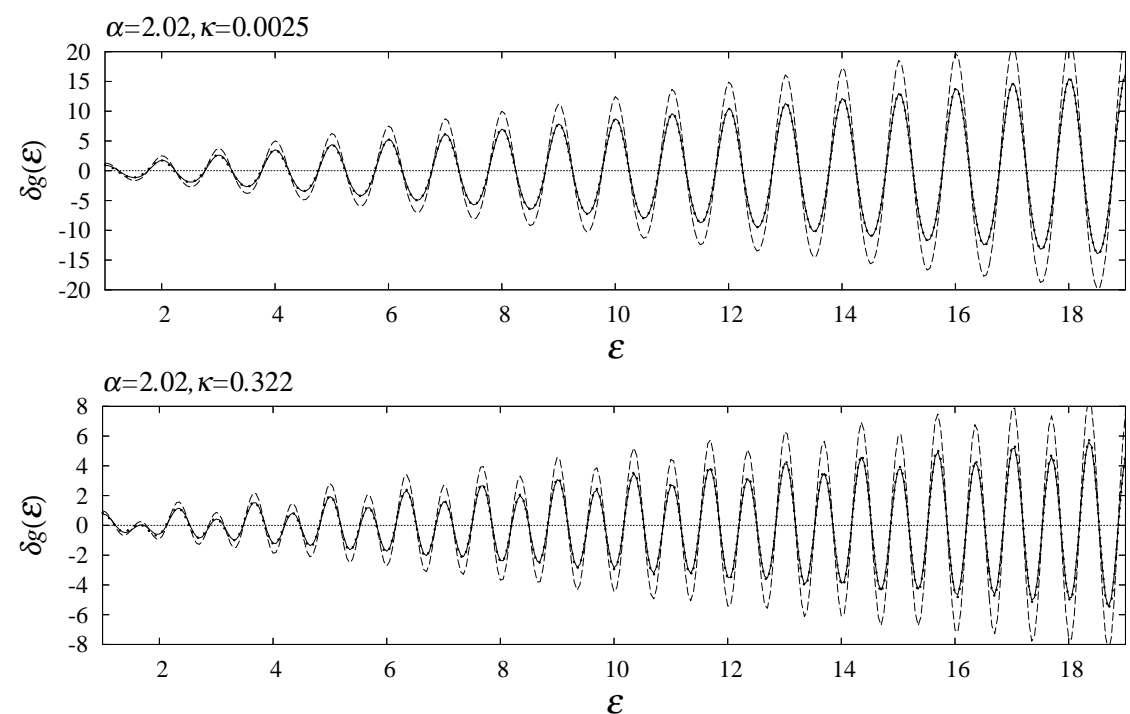

Figure 17. Comparison of the global and local uniform approximations. Solid, dashed and dotted curves represent uniform approximations UA2L, UA2 and QM, respectively, calculated at the bifurcation points of $(1,1)$ and $(2,1)$ bridge orbits. The periodic orbits included in the semiclassical level densities are same as in figure 15
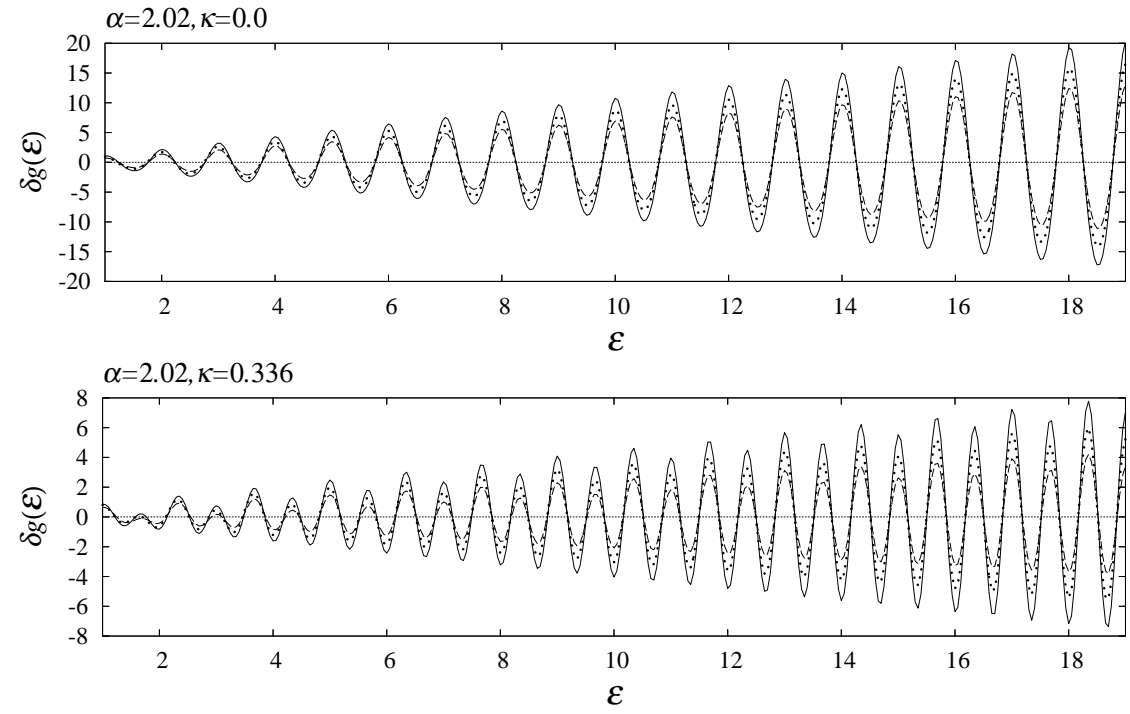

Figure 18. Same as figure 17 but calculated at the middle of two bifurcation points of $(1,1)$ and $(2,1)$ bridge orbits. 
mechanical coarse-grained level densities is very satisfactory.

We expect that the remaining differences and the slight variations of the normal form parameters can be reduced by including more terms in the normal form. This would be at the cost of losing the simple analytical forms of the uniform approximations and of having to determine more parameters numerically.

The exploration of suitable normal forms for bridge-orbit bifurcations in nonintegrable systems, such as those shown in figures 7 and 9, is the subject of further studies. The uniform approximations for non-integrable system form also an important subject for understanding the deformed shell structure in realistic nuclear mean-field models [22, 23].

\section{Acknowledgments}

K A acknowledges financial support by the Deutsche Forschungsgemeinschaft (graduate college 638 "Nonlinearity and nonequilibrium in condensed matter").

\section{Appendix A. Derivation of the mapping for bridge orbit bifurcations}

In this appendix, we derive a mapping from the Poincaré variables $(q, p)$ to action-angle variables $(\varphi, I)$ that are suitable for the normal forms of bridge orbit bifurcations. Let us consider the isotropic two-dimensional harmonic oscillator

$$
H=\frac{1}{2}\left(p_{x}^{2}+p_{y}^{2}\right)+\frac{1}{2} \omega^{2}\left(x^{2}+y^{2}\right) .
$$

All its trajectories are periodic with period $T=2 \pi / \omega$; they are ellipses which may degenerate to a circle or to linear librations. We parameterize these periodic orbits using the two constants of motion energy $E$ and angular momentum $L=x p_{y}-y p_{x}$. The orbit whose longer semiaxis lies on the $x$ axis is written as

$$
\begin{aligned}
& x_{0}(t)=q_{c}\left(\cos \frac{\theta}{2}+\sin \frac{\theta}{2}\right) \cos (\omega t), \\
& y_{0}(t)=q_{c}\left(\cos \frac{\theta}{2}-\sin \frac{\theta}{2}\right) \sin (\omega t), \\
& q_{c}=\sqrt{L_{c} / \omega}, \quad L_{c}=E / \omega, \quad L=L_{c} \cos \theta .
\end{aligned}
$$

Here $L_{c}$ is the maximum angular momentum at fixed energy, which is that of the circular orbit running anti-clockwise in the $(x, y)$ plane.

Rotating this orbit by an angle $\varphi / 2$ about the origin, one obtains the most general orbit

$$
\begin{aligned}
& x(t)=x_{0}(t) \cos \frac{\varphi}{2}-y_{0}(t) \sin \frac{\varphi}{2}, \\
& y(t)=x_{0}(t) \sin \frac{\varphi}{2}+y_{0}(t) \cos \frac{\varphi}{2}, \quad 0 \leq \varphi \leq 2 \pi .
\end{aligned}
$$

The projected Poincaré surface of section $\Sigma:=\left\{\left(x\left(t_{i}\right), p_{x}\left(t_{i}\right)\right) \mid y\left(t_{i}\right)=0, \dot{y}\left(t_{i}\right)>0\right\}$ at successive times $(i=1,2, \ldots)$, with $t_{i+1}=t_{i}+T$, defines the Poincaré variables $(q, p)=\left(x\left(t_{i}\right), p_{x}\left(t_{i}\right)\right)$. Each point $(q, p)$ corresponds to an orbit with period $T$, and therfore the Poincaré variables $(q, p)$ have a one-to-one correspondence with the variables $(\theta, \varphi)$, given by

$$
q=\frac{q_{c} \cos \theta}{\sqrt{1-\sin \theta \cos \varphi}}, \quad p=\frac{p_{c} \sin \theta \sin \varphi}{\sqrt{1-\sin \theta \cos \varphi}}, \quad p_{c}=\omega q_{c} .
$$


The Jacobian of the transformation $(\varphi, \theta) \rightarrow(q, p)$ is

$$
\frac{\partial(q, p)}{\partial(\varphi, \theta)}=-\frac{1}{2} p_{c} q_{c} \sin \theta
$$

We now define the action variable $I$ by

$$
I=\rho \cos \theta, \quad \rho=\frac{1}{2} p_{c} q_{c}=\frac{1}{2} L_{c}, \quad-\rho \leq I \leq \rho .
$$

Comparing to (A.4), one sees that $I=L / 2$. One also sees easily that the transformation $(\varphi, I) \rightarrow(q, p)$ becomes canonical since

$$
\frac{\partial(q, p)}{\partial(\varphi, I)}=1 .
$$

For the inverse transformation, one finds from (A.6) and (A.8) the relation

$$
I=\frac{1}{2} q \sqrt{4 \rho \omega-p^{2}-\omega^{2} q^{2}}, \quad \frac{1}{2}\left(p^{2}+\omega^{2} q^{2}\right)<2 \rho \omega(=E) .
$$

\section{References}

[1] Gutzwiller M C 1971 J. Math. Phys. 12343

[2] Balian R and Bloch C 1972 Ann. Phys. (N. Y.) 6976

[3] Cvitanović P (ed) 1992 Chaos Focus Issue on Periodic Orbit Theory, Chaos 2 pp 1-158

[4] Gutzwiller M C 1990 Chaos in classical and quantum mechanics (New York: Springer-Verlag)

[5] Stöckmann H-J 1999 Quantum Chaos: an Introduction (Cambridge: Cambridge University Press)

[6] Haake F 2001: Quantum Signatures of Chaos (New York: Springer-Verlag, 2nd edition)

[7] Brack M and Bhaduri R K 2003 Semiclassical Physics revised edn (Boulder, CO: Westview Press)

[8] Brack M and Jain S R 1995 Phys. Rev. A 51, 3462

[9] Sieber M 1997 J. Phys. A: Math. Gen. 304563

[10] Ozorio de Almeida A M and Hannay J H 1987 J. Phys. A: Math. Gen. 205873

[11] Ozorio de Almeida A M 1988 Hamiltonian systems: Chaos and quantization (Cambridge: Cambridge University Press)

[12] Meyer K R 1970 Trans. Amer. Math. Soc. 14995

[13] Sieber M 1996 J. Phys. A: Math. Gen. 294715

[14] Schomerus H and Sieber M 1997 J. Phys. A: Math. Gen. 304537

[15] Sieber M and Schomerus H 1998 J. Phys. A: Math. Gen. 31165

[16] Schomerus H 1997 Europhys. Lett. 38423 Schomerus H and Haake F 1997 Phys. Rev. Lett. 791022 Schomerus H 1998 J. Phys. A: Math. Gen. 314167

[17] Kaidel J and Brack M 2004 Phys. Rev. E 70 016206; ibid 72049903 (Erratum)

[18] Brack M and Tanaka K 2008 Phys. Rev. E 77046205

[19] Creagh S C 1996 Ann. Phys. (N. Y.) 24860

[20] Tomsovic S, Grinberg M and Ullmo D 1995 Phys. Rev. Lett. 754346

[21] Brack M, Meier P and Tanaka K 1999 J. Phys. A: Math. Gen. 32331 Brack M, Ögren M, Yu Y and Reimann S M 2005 J. Phys. A: Math. Gen. 389941

[22] Arita K 2004 Int. J. Mod. Phys. E 13191

[23] Arita K 2006 Phys. Scr. T 12514

[24] Eriksson A B and Dahlqvist P 1993 Phys. Rev. E 47 1002; see also the appendix of [18]

[25] Brack M, Fedotkin S N, Magner A G and Mehta M 2003 J. Phys. A: Math. Gen. 361095

[26] Pletyukhov M and Brack M 2003 J. Phys. A: Math. Gen. 369449

[27] Golubitsky M and Schaeffer D G 1985 Singularities and Groups in Bifurcation Theory (New York: Springer-Verlag)

[28] Poston T and Stewart I N 1978 Catastrophe Theory and its Applications (London: Pitman)

[29] de Aguiar M A M, Malta C P, Baranger M, and Davies K T R 1987 Ann. Phys. (NY) 180167

[30] Berry M and Tabor M 1976 Proc. Roy. Soc. Lond. A 349101

[31] Abramowitz M and Stegun I A 1970 Handbook of Mathematical Functions (New York: Dover Publications, 9th printing) 